\title{
Bülent İplikşioğlu
}

\section{Ländliche Siedlungen und das Territorium von Termessos (Pisidien)*}

Die Ruinen von Termessos ${ }^{1}$ in Südwest-Pisidien bzw. in West-Pamphylien nach der Provinzeinteilung des Hierokles ${ }^{2}$ erheben sich $24 \mathrm{~km}$ nordwestlich des heutigen Antalya (Attaleia) in einer Seehöhe von über $1000 \mathrm{~m}$ am Südwest-Fuß des Güllük bzw. Güldere Dağı (Solymos; 1265 m) $)^{3}$. Ursprünglich zwar kein griechischer Stadtstaat, durch den starken Hellenisierungsvorgang jedoch in Form einer griechischen Polis organisiert, hatte auch Termessos ein Territorium, d.h. einen Herrschaftsbereich bzw. Landbesitz. Das Territorium ist in TAM III,1, Nr. 6 als

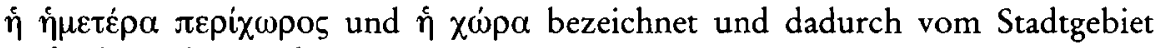
(= $\dot{\eta} \pi$ ó $\lambda$ ıs) abgesondert.

In TAM III,1, Nr. 104 aus dem 3. Jahrhundert n.Chr. werden zwei Verwaltungsbezirke in der Chora von Termessos genannt, für deren Schutz eigene, auch

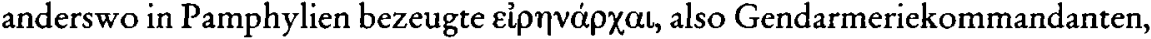

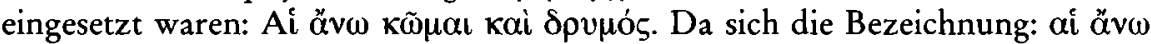

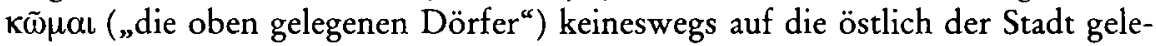
gene Ebene, sondern auf im Vergleich mit dem termessischen Stadtgebiet höhere Berge mit entsprechendem Platz für mehrere Siedlungen bezogen haben kann,

\footnotetext{
* Abkürzungsverzeichnis anhängend, S. $124 \mathrm{f}$.

1 Türkei-Karte 1:200000, Harita Genel Müdürlüğü, H IV: Elmalı (Ankara 1943 [1951]) IIb/ 39; R. Heberdey, in: RE V A,1 (1934) 734, s.v. „Termessos, Nr. ${ }^{4}$.

2 Zur Grenze zwischen Lykien und Pamphylien vgl. TAVO, B V 15. 2: Lykien und Pamphylien; Sh. Jameson, in: RE Suppl. XIII (1973) 268-271, s.v. "Lykia" und W. Ruge, in: RE XVIII,3 (1949) 354-358, s.v. „Pamphylia“.

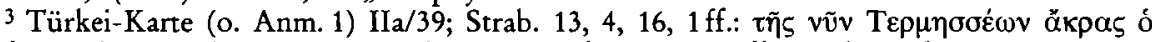

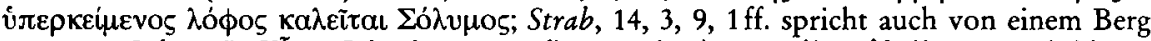

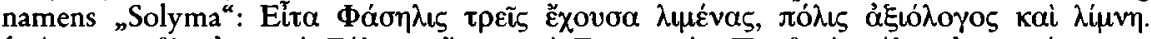

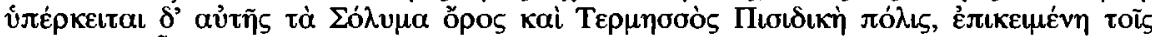

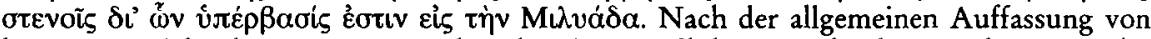
heute entspricht der erstere Berg dem heutigen Güllük Dağı, der letztere hingegen der Gebirgskette an der ostlykischen Küste, die vom Kap Chelidonia über die Berge Phoinikus und Olympos (Musa Dağı) zum Berg Solymos verläuft (Tahtalı Dağı), vgl. K. G. Lanckoroński, Städte Pamphyliens und Pisidiens, II: Pisidien (Prag, Wien, Leipzig 1892) 2, Anm. 1; W. Ruge, in: RE III A,1 (1927) 988f., s.v. " $\Sigma o ́ \lambda u \mu \alpha^{“}$; D. Magie, Roman rule in Asia Minor (Princeton 1950) 517ff.; 1136, Anm. 11; O. Akşit, Likya Tarihi (İstanbul 1967) $21 \mathrm{f}$.
} 
setzte Heberdey ${ }^{4}$ die im bis zu einer Höhe von $3000 \mathrm{~m}$ ansteigenden Bergland südwestlich von Termessos gelegene und von R. Paribeni und P. Romanelli ${ }^{5}$ ent-

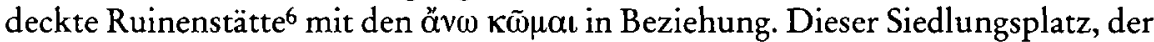
über eine Ummauerung und eine Agora sowie zwei Nekropolen verfügt, wird

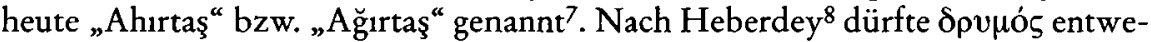
der als Eigenname oder als Appellativum angesehen werden und ist in der Nähe

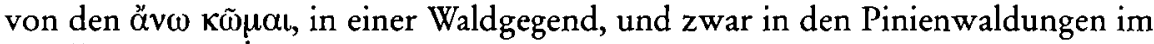
Quellgebiet des İncirlisu Çayı, nordwestlich von Termessos (und südsüdöstlich des Çandır Dağı), zu suchen, die die alte Straße von Attaleia nach Isinda durchzieht. Dieser Bezirk (oder Ort?) umfaßte vermutlich auch die Gegend des Yenice Boğazı ( $\tau \dot{\alpha} \Sigma \tau \varepsilon v a ́)$, einer Schlucht des Kuruçay, nordöstlich von Termessos ${ }^{9}$. Heberdey ${ }^{10}$ nahm neben den $\alpha$ $v \omega \kappa \tilde{\omega} \mu \alpha \iota$ und $\delta \rho v \mu o ́ \varsigma$ mindestens noch einen dritten Verwaltungsbereich in der östlich der Stadt gelegenen Ebene an, da dort, in Evdir Hanı, nördlich von Uzunkuyu Kahvesi ${ }^{11}$, die Tätigkeit von Eirenarchen

\footnotetext{
4 Termessische Studien (Wien, Leipzig 1929) 6.

${ }^{5}$ Studi e ricerche archeologiche nell'Anatolia meridionale, in: MonAL 23 (1914) 186-202.

${ }^{6} 10 \mathrm{~km}$ südlich von Termessos, $23 \mathrm{~km}$ westlich von Antalya; Türkei-Karte (o. Anm. 1) IIc/ 39 (Dam).
}

7 Für diesen Ort vgl. auch Ch. Schuler, Ländliche Siedlungen und Gemeinden im hellenistischen und römischen Kleinasien (München 1998) 48.

8 (o. Anm. 4) 11.

9 Etwa 22-28 km nordwestlich von Antalya (Türkei-Karte [o. Anm. 1] IIa/39); für die Erwähnung dieses Passes mit einer zur Außenfortifikation von Termessos gehörigen Festung anläßlich des Alexanderzuges vgl. Arr., anab. 1, 27, 8; Strab. 666; vgl. auch J. Seibert, Die Eroberung des Perserreiches durch Alexander den Großen auf kartographischer Grundlage (Wiesbaden 1985) 54. Für $\delta \rho v \mu o ́ \varsigma s$ s. auch Heberdey (o. Anm. 1) 766; St. Mitchell, Anatolia: Land, men, and gods in Asia Minor, I: The Celts in Anatolia and the impact of Roman rule (Oxford 1993) 196; Schuler (o. Anm. 7) 115 mit Anm. 78.

10 (o. Anm. 4) 11 und (o. Anm. 1) 766.

$1117 \mathrm{~km}$ nordwestlich von Antalya; Türkei-Karte (o. Anm. 1) IIa/40. Daß die Ruinen beim Evdir Hanı "Lagon“ hießen (vgl. Türkei-Karte (o. Anm. 1) IIa/39 [Lagonyaharabesi]), ist eine aus den inschriftlich genannten $\lambda \alpha$ yóves (Heberdey [o. Anm. 4] 8; ders., TAM, III: Tituli Pisidiae; 1: Tituli Termessi et Agri Termessensis [Wien 1941] Nr. 908) resultierende irrige Annahme (Ü. Serdaroğlu, in: PECS, 477, s.v. „Lagon“). Die Siedlung führte vielleicht ursprünglich, als eine $\kappa \omega ́ \mu \eta \eta$ in der Chora von Termessos, den Namen „Anydros“ und später, als Neugründung des 5. Jahrhunderts n.Chr. und wie Eudokias in Lykien vermutlich auch nach Eudokia, der Gattin Kaiser Theodosius' II., benannt, „Eudokias“, vgl. Heberdey (o. Anm. 4) 8. 14f; ders. (o. Anm. 1) 734. 736 f. 750 f.; Heberdey, TAM (o.) Nr. 779 und ebd. 271; L. Robert, Noms indigènes dans l'Asie-Mineure gréco-romaine, I (Paris 1963) 140; G. E. Bean, Turkey's southern shore: An archaeological guide (London 1968) 113; mit dem neuen Namen als Stadt der spätantiken Provinz Pamphylien bei Hier. verzeichnet (680, 2b); Bischof Timotheos von Termessos und Eudokias nahm im Jahre 431 am Konzil von Ephesos teil (ACO, I 1, 2 S. 63, Nr. 185; I 1, 3 S. 19; I 1, 7 S. 114, Nr. 84) und im Jahre 448 Bischof Sabinianos von Termessos, Eudokias und Iobia an der Synode von Konstantinopel (vgl. unten Anm. 112; vgl. auch $H$. Brandt, Gesellschaft und Wirtschaft Pamphyliens und Pisidiens im Altertum [Bonn 1992] 177); daß der Brief des Metropoliten von Perge an Kaiser Leon I. im Jahre 458 von Auxentius Termissae und Innocentius Eudociados unterschrieben wurde (ACO, II 5, 60), zeigt, daß damals Eudokias bereits die Unabhängigkeit von Termessos erreicht hatte. Eudo- 


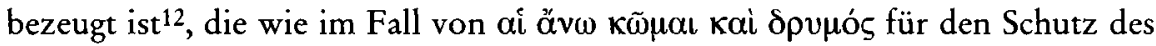
Bezirkes eingesetzt waren. In einer 1993 von uns nahe dem West-Eingang des ter-

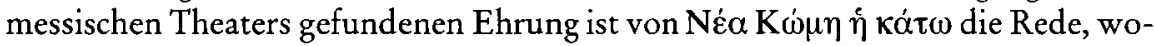
bei wir annehmen, daß mit dem Zusatz $\dot{\eta}$ Kót $\omega$ gemäß der geographischen Situation das Gegenstück zu den wohl in den Bergen westlich von Termessos gelegenen

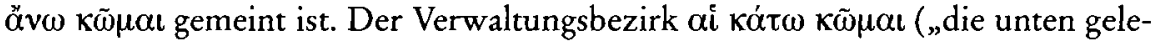
genen Dörfer") umfaßte also die im Osten der Stadt vorgelagerte Ebene, und wie

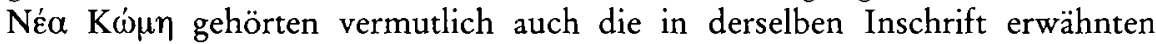

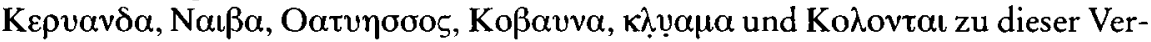
waltungseinheit.

Da die Grenzen des Territoriums in hellenistischer Zeit angesichts der unzureichenden Quellenlage einstweilen nicht ganz feststellbar waren, stellte Heberdey diesbezüglich keine Vermutung an; er war nur der Meinung, daß sich die Stadt in ihrer ganzen Geschichte in Richtung Westen am meisten im 3. Jahrhundert v. Chr. ausgebreitet und deshalb auch ihre innerhalb der Polis Oinoanda nordwestlich oberhalb von Incealiler ${ }^{13}$ mit eigener bule und gerusia gelegene Kolonie „Klein-

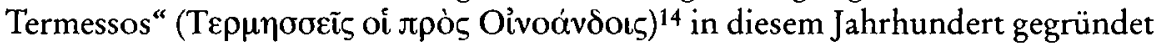
haben müsse ${ }^{15}$. Da die Inschriften der Römerzeit aber reichlicher sind und zugleich in diesem Zeitalter am wenigsten Grenzänderungen erfolgt waren, hat Heberdey einen gründlichen Versuch zur Festlegung des termessischen Territoriums gemacht ${ }^{16}$ : Er nahm an, daß die Chora von Termessos bis zu den Quellen des Karaman Çayı hinauf reichte. Die natürlichen Grenzen dieses Gebietes bilden im Süden und Südwesten das Gelände zwischen dem Karaman Çayı und dem Alakır Çayı, im Westen die Bergkette, die, im Süden mit dem İskenderköy Dağı beginnend, im Norden beim İstanoz Çayı (Bozçay, Akkırca Çayı; Tauros) den höchsten Punkt (Çandır Dağı) erreicht, und im Osten die Erhebungen, die die Quellgebiete des Karaman Çayı und des Çandır Çayı voneinander trennen. Nach Heberdey stellt die natürliche Grenze in der östlich der Stadt gelegenen Ebene die mittlere pamphylische Terrasse dar, wenn der Herrschaftsbereich von Termessos die bereits genannte Siedlung beim Evdir Hanı mit ihrem Landbesitz einschloß. Dementsprechend verlief die Ost-Grenze westlich der $\Lambda v \rho \beta \omega \tau \tilde{\omega} v \kappa \omega \dot{\mu} \mu$ (Pazargediği

kias erscheint wie Termessos selbst bis in das 12. Jahrhundert als eigenes Bistum in den Notitiae episcopatuum.

12 Heberdey, TAM (o. Anm. 11) Nr. 906. 908f. 912-915; vgl. auch L. Robert, Études anatoliennes: Recherches sur les inscriptions grecques de l'Asie Mineure (Paris 1937) 105; Mitchell (o. Anm. 9) 196 mit Anm. 251.

13 Türkei-Karte (o. Anm. 1) IIç/31.

14 Steph. Byz. 618; Eusth., comm. Dion. 368; J. J. Coulton, Termessians at Oinoanda, in: AS 32 (1982) 115-131; $M$. Wörrle, Stadt und Fest im kaiserzeitlichen Kleinasien (München 1988) 45-53; C. F. Eilers, N. P. Milner, Q. Mucius Scaevola and Oenoanda: A new inscription, in: AS 45 (1995) 85.

15 Heberdey (o. Anm. 1) 734.

16 Heberdey (o. Anm. 4) 5-15; ders. (o. Anm. 1) 734-737; vgl. auch die Karte bei ders. (o. Anm. 4) 5, Fig. 1 und wiederholt in Heberdey, TAM (o. Anm. 11) 1. 
oder Örenkale) $1 \mathrm{~km}$ nördlich von Varsak ${ }^{17}$, da diese Ruinenstätte nach Ausweis ihrer Inschriften ${ }^{18}$ der Nachbarstadt Perge gehörte. Nach Heberdey ist neben der ganz hypothetischen West-Grenze auch die Nord-Grenze schwer zu bestimmen: Die für die Begrenzung im Norden wichtige, im etwas südlich des Çubuk Boğazı gelegenen Kırkgöz Hanı vermauerte Grabinschrift ${ }^{19}$, in der die Grabbuße ... kai

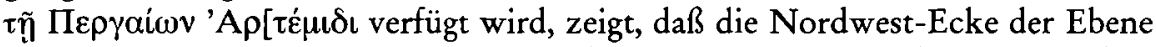
zum Gebiet von Perge gehörig war, und die Grenze noch südlicher, nahe dem oben erwähnten Yenice Boğazı ( $\tau \dot{\alpha} \Sigma \tau \varepsilon v \alpha \dot{)}$ gezogen werden muß. Danach umfaßte das termessische Territorium ungefähr ein Dreieck von 550 bzw. 600 qkm Fläche, im wesentlichen den Ober- und Mittellauf des Incirlisu Çayı und des Karaman Çayı und ca. 100 bzw. 150 qkm vom Westen der mittleren pamphylischen Terrasse.

Neue Untersuchungen ${ }^{20}$ haben jedoch erwiesen, daß in erster Linie die von Heberdey für die römische Zeit konstruierte West- und Süd-Grenze der termessischen Chora beträchtlich erweitert werden muß:

Mitchell publizierte eine Inschrift ${ }^{21}$, die von French während eines Surveys $1990 \mathrm{im} 10 \mathrm{~km}$ östlich von Korkuteli, $15 \mathrm{~km}$ nordwestlich von Termessos und $40 \mathrm{~km}$ nordwestlich von Antalya gelegenen Dorf Bayat ${ }^{22}$ ungefähr zwischen Termessos und Isinda $a^{23}$ gefunden wurde. Der Stein stammt ursprünglich aus einem Feld südlich des ca. $1 \mathrm{~km}$ im Süden des Dorfes, auf der rechten Seite des Tauros

$1725 \mathrm{~km}$ östlich von Termessos, $12 \mathrm{~km}$ nördlich von Antalya, $9 \mathrm{~km}$ westnordwestlich von Perge (Türkei-Karte 1:200000, Harita Genel Müdürlüğü, H V: Antalya [Ankara 1944 (1950)] IIa/41); vgl. Heberdey (o. Anm. 4) 9; R. Merkelbach, S. Şabin, Die publizierten Inschriften von Perge, in: EA 11 (1988) 158 ff., Nr. 152; Brandt (o. Anm. 11) 127; S. Şabin, Studien zu den Inschriften von Perge II: Der Gesandte Apollonios und seine Familie, in: EA 25 (1995) $2 \mathrm{f}$. 12 ff.; Schuler (o. Anm. 7) 262 (Anm. 307). 279ff.

18 H. A. Ormerod, E. S. G. Robinson, Notes and inscriptions from Pamphylia, in: ABSA 17 (1910/1911) 225 ff., Nr. 1, Z. 3-5; Nr. 8, Z. 11; Nr. 9, Z. 2.

19 B. Pace, La zona costiera da Adalia a Side, in: ASAA 3 (1916/1920 [1921]) 35, Nr. 19.

20 St. Mitchell, Termessos, king Amyntas and the war with the Sandaliôtai: A new inscription from Pisidia, in: D. French (Hrsg.), Studies in the history and topography of Lycia and Pisidia in memoriam A. S. Hall (Ankara, Oxford 1994) 95-105 mit Bildtafeln 6.1-2; D. French, Isinda and Lagbe, ebd. $73 \mathrm{f}$. mit Tafeln 4.1-3; J. Nollé, Kitanaura: Münzen und Geschichte einer kleinen Stadt in den ostlykischen Bergen, in: JNG 46 (1996) 7-29; M. Zimmermann, Probus, Carus und die Räuber im Gebiet des Pisidischen Termessos, in: ZPE 110 (1996) 265-277; B. İplikçioğlu, Zum Territorium von Termessos in Pisidien, in: P. Scherrer, H. Taeuber, H. Thür (Hrsg.), Steine und Wege. Festschrift für Dieter Knibbe zum 65. Geburtstag (Wien 1999) 309-314.

21 D. French, The year's work, in: AS 41 (1991) 11; St. Mitchell, Amyntas in Pisidien Der letzte Krieg der Galater, in: AMS 12: Forschungen in Galatien (1994) 97-103; ders. (o. Anm. 20); vgl. auch ders., Native rebellion in the Pisidian Taurus, in: $K$. Hoproood (Hrsg.), Organised crime in antiquity (London 1998) 164 mit Anm. 33.

22 Türkei-Karte (o. Anm. 1) Ila/37; vgl. auch A. Scbönborn bei C. Ritter, Die Erdkunde im Verhältnis zur Natur und zur Geschichte des Menschen usw., 19. Theil: Klein-Asien, II (Berlin 21859) 684.

${ }^{23}$ Für Isinda vgl. G. E. Bean, in: PECS, 417, s.v. „Isinda“; L. Zgusta, Kleinasiatische Ortsnamen (Heidelberg 1984) $202 \$ 384-1$; French (o. Anm. 21) 11; ders. (o. Anm. 20) 57 f.; D. Stiernon, in: DHGE, Fasc. 150 (1995) 228-230, s.v. „Isinda“. 
(Korkuteli Çayı) befindlichen und heute „Bayat Hüyük“ genannten prähistorischen Hügels mit hellenistisch-römischer Keramik und antiken Werksteinen so-

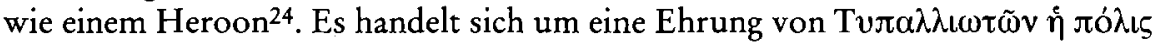

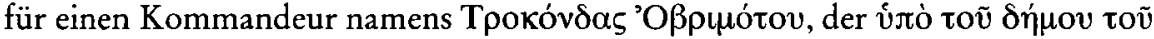

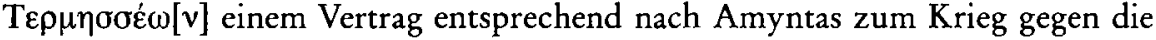
Sandaliotai entsandt wurde, vieles Gedenkenswerte durchgeführt hat, bei heroischen Kriegstaten gestorben ist und vom König selbst wegen seiner Tapferkeit mit den größten Beweisen seines Heroismus und mit einer vergoldeten Statue geehrt wurde.

Der 39 v. Chr. von M. Antonius als König der Pisidier eingesetzte galatische Tetrarch Amyntas, der 36 v. Chr. auch die Herrschaft über Galatien, Lykaonien und Teile von Pamphylien übernahm ${ }^{25}$, führte harte Kriege im Taurusgebirge, während deren er Kremna erobern konnte, nicht aber Sandalion ( $\Sigma \alpha v \delta \alpha \dot{\lambda} \lambda$ เov, heute Sandal Asarı), eine Festung an der Einmündung des Çukurdere in den Isparta Çay1 (ein Quellfluß des Kestros), zwischen Kremna und Sagalassos ${ }^{26}$. Mitchell nahm an, daß der geehrte Trokondas ein Termessier war, da dieser von den Termessiern zu Amyntas geschickt wurde, und daß der Stammort des Denkmales sowohl den Landbesitz als auch den Beerdigungsplatz des Trokondas darstellte, obwohl die Bestattung mit ausländischer Ehrung gefeiert wurde. Die Inschrift erwähnt also die Allianz zwischen dem galatischen König Amyntas und Termessos im Krieg gegen Sandalion, wobei der Druck auf die Sandalioten offensichtlich von termessischen Truppen ausgeübt worden war. Die in die Zeit um 30 v. Chr. datierende Inschrift rückt somit auch den bis dahin nur vermuteten Status von Termessos unter dem König Amyntas ins Licht.

Auf Grund der Annahme Mitchells, daß Trokondas ein Termessier war und in seinem Landbesitz beerdigt wurde, wo ihm die Typallioten das bereits genannte Denkmal gesetzt haben, ist die Nordwest-Grenze der termessischen Chora noch westlicher als in Heberdeys Vorschlag für die römische $\mathrm{Zeit}^{27}$, d.h. auch den Fundort umfassend, anzusetzen. (Ein aus dem Dorf Bayat stammendes, jetzt im Depot in Korkuteli befindliches Fragment einer Inschrift mit Erwähnung eines

${ }^{24}$ A. M. Woodward, H. A. Ormerod, A journey in South-Western Asia Minor, in: ABSA 16 (1909/1910) 90. 126; French (o. Anm. 20) 74.

${ }^{25} \mathrm{H}$. Buchbeim, Die Orientpolitik des Triumvirn M. Antonius (Göttingen 1960) $56 \mathrm{f}$.

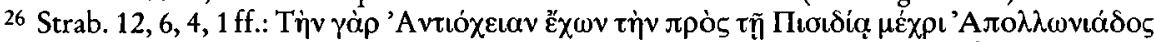

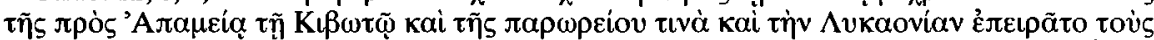

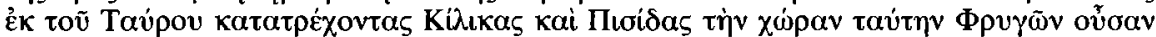

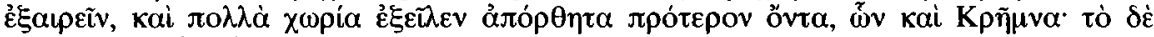

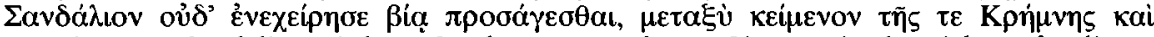

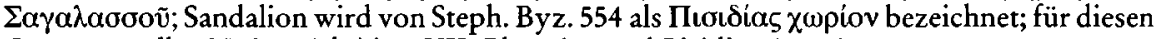
Ort s. K. Belke, N. Mersich, TIB, VII: Phrygien und Pisidien (1990) 371.

${ }^{27}$ Heberdey (o. Anm. 4) 5, Fig. 1 hat die West-Grenze entlang der Ost-Seite des Tales des Istanoz Çayı (Bozçay, ant. Tauros)) gezogen und somit Bayat ausgeschlossen. Dazu Mitchell (o. Anm. 20) 98: "There is no reason, however, why the border between Termessos and Isinda should not have run along the west side of the valley, making an important and fertile addition to 'Termessos' territory ...". 
Eirenarchen [L86] bestätigt auch die Annahme, daß die Gegend von Bayat zur Chora von Termessos gehörte. Bei diesem Eirenarchen handelt es sich wohl um den Gendarmariekommandanten, der für den dortigen Verwaltungsbezirk im Territorium, also höchstwahrscheinlich für $\delta \rho v \mu o ́ s$, den Heberdey im Waldgebiet nordwestlich der Stadt zu suchen versuchte, zuständig war). Wegen dieser Inschrift rechnet auch French ${ }^{28}$ die beiden Seiten des Bozova- bzw. Bozçay-Tales nördlich vom $5 \mathrm{~km}$ südsüdwestlich von Bayat, $16 \mathrm{~km}$ westnordwestlich von Termessos gelegenen Dorf Yazır zum Territorium von Termessos. Es ist akzeptabel, daß die Nordwest-Grenze schon seit hellenistischer Zeit entlang der westlichen Seite des Tales des İstanoz Çayı (Bozçay) verlief ${ }^{29}$.

In der $10 \mathrm{~km}$ südwestlich von Yazır, im Gebiet des dem Dorf Sımandır zugehörigen Geländes Sımandır Susuzu gelegenen Flur Delikinler haben wir auf Naturfelsen die Grabinschrift eines Termessiers gefunden, die eindeutig für die Zugehö-

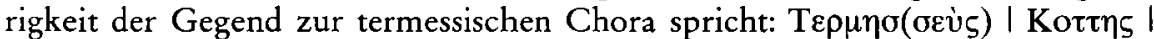
Máveov[s]. 3-4 km nördlich des $21 \mathrm{~km}$ südwestlich von Yazır und $33 \mathrm{~km}$ westsüdwestlich von Termessos befindlichen Dorfes Beyiş (auch Beğiş) ${ }^{30}$ liegt eine kleine hellenistisch-römische Ansiedlung auf einem Felshügel, die noch zum Territorium von Termessos gehörte ${ }^{31}$ (wohl der westlichste Ort in der Chora). Im $12 \mathrm{~km}$ südöstlich von Beyiş gelegenen Dorf Imecik ${ }^{32}$ am West-Hang der Bey Dağları gibt es ein Felsheiligtum des Kakasbos-Herakles ${ }^{33}$ und südöstlich des Dorfes eine antike Stätte (Asar) ${ }^{34}$, die innerhalb der termessischen Chora liegen ${ }^{35}$.

In der Ruinenstätte $3 \mathrm{~km}$ südwestlich des $7 \mathrm{~km}$ südwestlich von İmecik gelegenen Dorfes Ovac1k ${ }^{36}$ wurde im Jahre 1975 eine auf vier Seiten beschriftete Stele

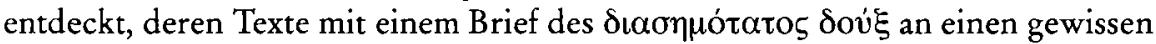

28 (o. Anm. 20) 57 mit Tafeln 4.1-3.

29 Die im Hüyük Mahallesi des Dorfes Yazır $(1 \mathrm{~km}$ nördlich von Yazır, $3,5 \mathrm{~km}$ südwestlich von Bayat, $16 \mathrm{~km}$ westnordwestlich von Termessos [Türkei-Karte (o. Anm. 1) IIa/37]) gefundenen Meilensteine aus diokletianisch-konstantinischer Zeit geben Termessos als Anfang der Straße an; $A$. M. Woodward, Inscriptions from Western Pisidia, in: ABSA 17 (1910/1911) 207 f.; Heberdey, TAM (o. Anm. 11) Nr. 942-944; D. French, Roman roads and milestones of Asia Minor, Fasc. 2: An interim catalogue of milestones, Part 1 (Oxford 1988) Nr. 191. Hier auch ein Fragment eines Marmorquaders mit lateinischer Grabinschrift: Woodward, Ormerod (o. Anm. 24) 90. 126. $1 \mathrm{~km}$ südlich von Yazır befindet sich eine Ruinenstätte (TürkeiKarte [o. Anm. 1] IIa/37 [Hb.]) mit einem einzigen rock-cut-Sarkophag und einem kleinen prähistorischen Hügel. Nach French (o. Anm. 20) 74 war dieses Areal Teil der termessischen Chora (vgl. auch $L$. Robert, Hellenica: Recueil d'épigraphie, de numismatique et d'antiquités grecques, X [Paris 1955] 8, der die Gegend zum Territorium von Isinda rechnete), zu der auch ein niedriger Hügel südlich davon, ca. $1,9 \mathrm{~km}$ südlich des Dorfes, nahe dem West-Ende einer kleinen Ebene (Ĭgligüme in der Karte) mit einem kleinen Bau auf der Kuppe gehörte.

30 Türkei-Karte (o. Anm. 1) IIc/36.

31 French (o. Anm. 20) 74.

$3245 \mathrm{~km}$ westsüdwestlich von Antalya; Türkei-Karte (o. Anm. 1) IIc/37.

33 L. Robert, Documents d'Asie Mineure, in: BCH 107 (1983) 569.

${ }^{34}$ A. Philippson, Reisen und Forschungen im westlichen Kleinasien, I-V (Gotha 1910-1915)

Bl. 6 mit Erg.-Hft. V; Heberdey (o. Anm. 4) 5, Fig. 1.

${ }^{35}$ French (o. Anm. 20) 74.

36 Türkei-Karte (o. Anm. 1) IIç/36. 


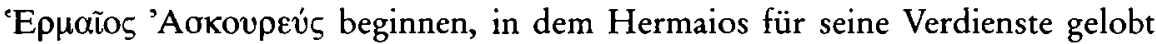

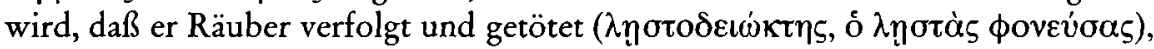

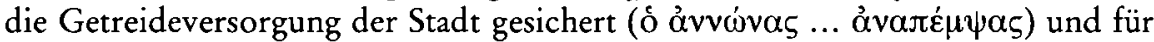
den Frieden gearbeitet hatte ${ }^{37}$. Dieser Hermaios war der Vater des M. Aủ $\rho$.

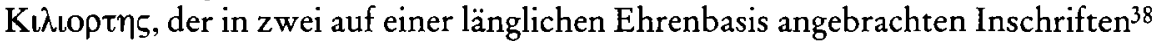
aus dem westsüdwestlich des Dorfes, $22 \mathrm{~km}$ ostnordöstlich von Elmalı gelegenen Askura $^{39}$, heute Söbüova mit Spuren einer alten Siedlung in der Umgebung ${ }^{40}$, erwähnt wird. Die auf der linken Seite des Monuments befindliche Inschrift ist

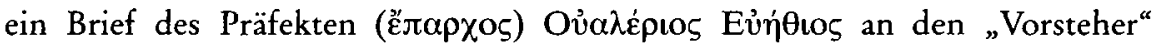

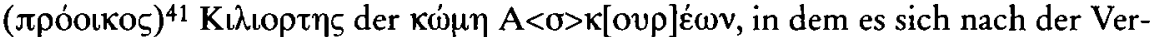
mutung Zimmermanns $s^{42}$ um die Anordnung der Festnahme der Räuberbanden ( $\sigma \dot{\sigma} \sigma \tau \eta \mu \alpha \tau \tilde{\omega} v \dot{\varepsilon} \pi \mid \pi 0 \lambda \alpha \sigma \alpha \dot{v \tau} \omega v$ ) handelt. In der anderen, auf der rechten Seite ein-

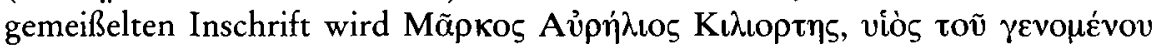

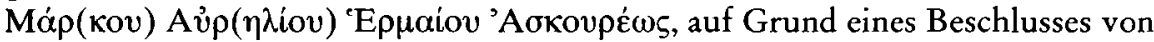

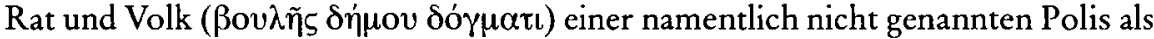

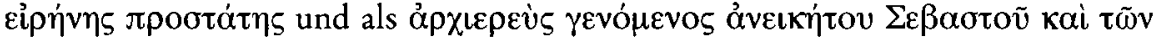

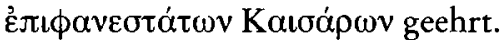

Wegen Inschriften, die die Anwesenheit von römischen Militärs im termessischen Gebiet für das ausgehende 3. Jahrhundert n.Chr. belegen ${ }^{43}$, wurde vermutet, daß wie im Falle Kremnas ${ }^{44}$ auch das termessische Territorium durch Über-

37 R. M. Harrison, Nouvelles découvertes romaines tardives et paléobyzantines en Lycie, in: CRAI (1979) 235-237; ders., Upland settlements in early medieval Lycia, in: Actes du Colloque sur la Lycie antique (Paris 1980) 112 ff.; SEG XXIX (1979) 1514; L. Robert, in: BE (1980) Nr. 488; B. İplikçioğlu, G. Çelgin, A. V. Çelgin, Neue Inschriften aus Nord-Lykien I (Wien 1992) $15 \mathrm{f} . ;$ C. Foss, Lycia in history, in: J. Morganstern (Hrsg.), The fort at Dereağzı and other material remains in its vicinity from antiquity to the middle ages (Tübingen 1993) 19f.; Zimmermann (o. Anm. 20) 265-269; Mitchell, Native rebellion (o. Anm. 21) 161 f.; G. H. R. Horsley, St. Mitchell, IK 57: The inscriptions of central Pisidia (Bonn 2000) 5 f., T.6; jetzt veröffentlicht von $M$. H. Ballance and Ch. Roueché, Appendix 2: Three inscriptions from Ovacik, in: $M$. Harrison, Mountain and plain from the Lycian coast to the Phrygian plateau in the late Roman and early Byzantine period, ed. by W. Young, Ann Arbor 2001, 89f. (= ,Ovacik I.A.i').

38 Íplikçioğlu, G. Celgin, A. V. Çelgin (o. Anm. 37) 12 f., Nr. 2; 14 ff., Nr. 4; vgl. auch SEG XLI (1991) 1390; C. Brixhe, A. Panayotou, in: BE (1993) Nr. 537; Zimmermann (o. Anm. 20) $267 \mathrm{ff}$. (ein ausgezeichneter Aufsatz zum Verständnis der Inschriften); Mitchell, Native rebellion (o. Anm. 21) 162 f.; jetzt veröffentlicht von Ballance, Roueché (o. Anm. 37) 96 f. (=, Ovacik II').

${ }^{39} \mathrm{Vgl}$. Harrison, Nouvelles découvertes (o. Anm. 37) 237; Íplikçioğlu, G. Çelgin, A. V. Celgin (o. Anm. 37) 15f.; Zimmermann (o. Anm. 20) 268; zur Namensform vgl. H. Hellenkemper, Fr. Hild, TIB, V: Kilikien und Isaurien (1990) s.v. "Askora“.

40 Íplikçioğlu, G. Çelgin, A. V. Çelgin (o. Anm. 37) 12.

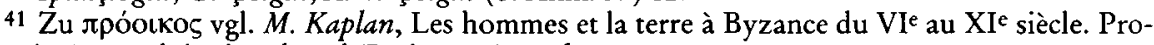
priété et exploitation du sol (Paris 1992) 198 f., 201.

42 (o. Anm. 20) 268.

43 Zimmermann (o. Anm. 20) 266, Anm. 8.

${ }^{44}$ Der Isaurer Palfuerius Lydius, der sich in Kremna verschanzt hatte, wurde 278 von Kaiser Probus mit Hilfe des Statthalters Terentius Marcianus besiegt; Hist. Aug., Probus 16, 4; 
griffe der Räuber in Mitleidenschaft gezogen worden ist ${ }^{45}$. Die Inschriften aus Ovacık und Söbüiova stellen nun eindeutige epigraphische Belege für die Existenz der Räuber in der Chora von Termessos dar. In dem Brief aus Söbüova war also die Rede von Räuberbanden, die die Kome im termessischen Herrschaftsbereich heimgesucht haben ${ }^{46}$, und die Ehrung für M. Aur. Kiliortes, der Kaiserpriester von Carus mit seinen Söhnen Carinus und Numerian (282-283 n. Chr.) $)^{47}$ gewesen

H. Brandt, Probus, Pacator Pamphyliae et Isauriae?, in: G. Bonamente, N. Duval (Hrsg.), Historiae Augustae Colloquium Parisinum (Macerata 1991) 91; Mitchell (o. Anm. 9) 234f.; J. Nollé, IK 43: Side im Altertum. Geschichte und Zeugnisse, 1: Geographie - Geschichte Testimonia - Griechische und Lateinische Inschriften (1-4) (Bonn 1993) 288, Anm. 28; St. Mitchell (mit S. Cormack, R. Fursdon, E. Owens und J. Öztürk), Cremna in Pisidia: An ancient city in peace and in war (London 1995) 177-218; Zimmermann (o. Anm. 20) 265 f.; St. Mitchell, The Pisidian survey, in: $R$. Mattberes (Hrsg.), Ancient Anatolia: Fifty years' work by the British Institute of Archaeology at Ankara (London 1998) $244 \mathrm{ff}$. mit Fig. 20.4;

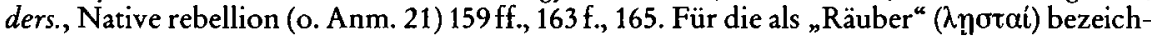
neten Isaurer, die wohl immer noch nicht endgültig in das Imperium Romanum eingegliederte selbständige Bergstämme waren, vgl. Zos., hist. 1, 59; K. Hopwood, Policing the hinterland: Rough Cilicia and Isauria, in: St. Mitchell (Hrsg.), Armies and frontiers in Roman and Byzantine Anatolia: Proceedings of a colloquium held at University College, Swansea, in April 1981 (Oxford 1983) 173-187; ders., Policing the Melas valley, in: Yayla 5 (1984) 25-29; ders., Towers, territory and terror: How the East was held, in: Ph. Freeman, D. Kennedy (Hrsg.), The defence of the Roman and Byzantine East: Proceedings of a colloquium held at the University of Sheffield in April 1986, I (Oxford 1986) 343-356; ders., Consent and control: How the peace was kept in Rough Cilicia, in: D. H. French, C. S. Lightfoot (Hrsg.), The eastern frontier of the Roman empire: Proceedings of a colloquium held at Ankara in September 1988, I (Oxford 1989) 191-201; Hellenkemper, Hild (o. Anm. 39) 18f.; K. Hopwood, The links between the coastal cities of Western Rough Cilicia and the interior during the Roman period, in: De Anatolia Antiqua I (Paris 1991) 305-309; Zimmermann (o. Anm. 20) 265; K. Tomaschitz, Unpublizierte Inschriften Westkilikiens aus dem Nachlaß Terence B. Mitfords (Wien 1998) 36, Anm. 94; 78 ff. Für die Errichtung eines Festungsgürtels gegen die Isaurer s. Not. Dign.: 61, Nr. XXIX; für die „Räuber“ im römischen Reich B. Shaw, Bandits in the Roman empire, in: P\&P 105 (1984) 3-51; in Lykien und Pamphylien F. Schindler, Die Inschriften von Bubon (Nordlykien) (Wien 1972) 15 f.; M. Sartre, L'Orient romain (Paris 1991) 290; in Pisidien: Mitchell, Native rebellion (o. Anm. 21).

45 Brandt (o. Anm. 44) 88; Zimmermann (o. Anm. 20) 266, Anm. 9; vgl. auch die Grabinschrift aus dem 3. Jahrhundert n.Chr., in der verfügt wird, daß die Grabbuße an den Demos

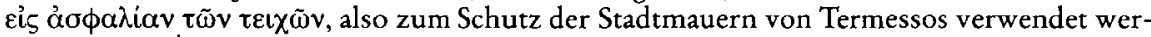
den solle: $B$. İplikçioğlu, G. Celgin, A. V. Celgin, Epigraphische Forschungen in Termessos und seinem Territorium II (Wien 1992) 19f., Nr. 9. Der dux in Ovacık, der den Namen Aủ]p. Oủpoi $\omega v$ trägt (s. Mitchell, Native rebellion [o. Anm. 21] 161), begegnet auch in einer Inschrift aus Arykanda (S. Şabin, IK 48: Die Inschriften von Arykanda [Bonn 1994] Nr. 26) in

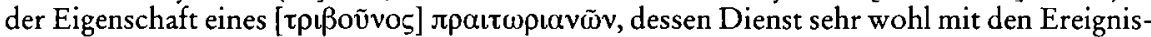
sen in Pisidien verbunden war, vgl. Mitchell ebd. 166; vgl. auch die Inschrift Heberdey, TAM (o. Anm. 11) Nr. 126 aus demselben Zeitraum, in der der $\mathfrak{\varepsilon} \xi$

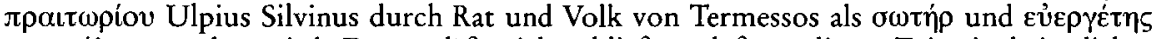

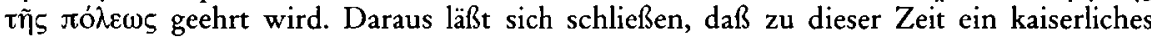
Hauptquartier in der Gegend anwesend war und daß sogar ein Gardepräfekt in die ernsthaften lokalen Unruhen im Gebiet von Termessos verwickelt wurde, Mitchell a.O.

46 Zimmermann (o. Anm. 20) 269.

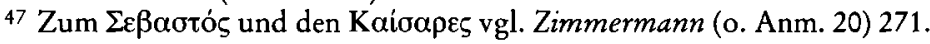


war, ist laut Zimmermann ${ }^{48}$ vermutlich durch die Polis Termessos gesetzt worden. Kiliortes und sein Vater Hermaios Askureus, der neben dem aktiven Einsatz gegen die Räuber auch für die Sicherung des Getreides für die Polis, d. h. für Termessos, sorgte, hatten als lokale Amtsträger mit der Verfolgung von Räubern zu tun, die mit den literarisch überlieferten Einfällen der Isaurer zur Zeit des Probus und noch des Carus (auf seinem im Frühjahr 283 erfolgten Zug in den O) in Pamphylien und in Teilen Lykiens zusammenhängen. Im fortgesetzten Kampf gegen die Räuber wurden im Gebiet von Termessos wahrscheinlich kleinere Einheiten eingesetzt, die in befestigten Plätzen auf der Chora stationiert wurden, wobei reguläre Truppen und lokale Einheiten vermutlich zusammengewirkt haben, da die auf dem Land eingesetzten Amtsträger der Polis, in erster Linie also die Eirenarchen ${ }^{49}$, die Lage nicht mehr kontrollieren konnten. Der in Askura begegnende Eparchos (Militärkommandant?) unterstand in diesem Kampf wohl dem in Inschriften aus der Ruinenstätte bei Ovacık, die noch keinen Namen hat, und Ter-

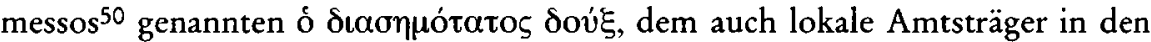
einzelnen komai an die Seite gestellt waren ${ }^{51}$. Eine der Festungen dieses $d u x$ war wohl die spätantike Festung im Südwesten des Dorfes Ovacik (Ovacık Asar1) ${ }^{52}$. Zimmermann ${ }^{53}$ nimmt an, daß es sich hier um ein auf der termessischen Chora angelegtes phrourion zum Schutze der ländlichen Bevölkerung handelt, die in der Kaiserzeit zunächst verstreut lebte und nun in den gefährlichen Zeiten zur eigenen Sicherheit in einer Ortschaft zusammengezogen und geschlossen angesiedelt wurde. Daß in Ovacık termessische Münzen gefunden wurden, spricht auch für die Zugehörigkeit des Kastells zur termessischen Chora ${ }^{54}$. Das Dorf Askura in

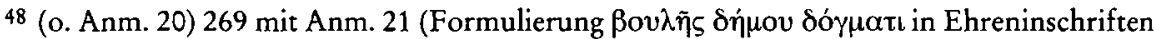
von Termessos: Heberdey, TAM [o. Anm. 11] Nr. 58. 80. 82. 122. 123. 138).

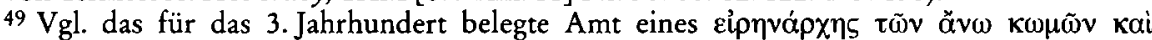

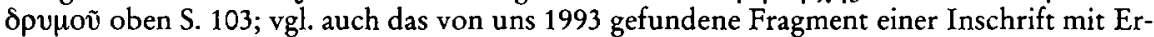
wähnung eines Eirenarchen aus dem $15 \mathrm{~km}$ nordwestlich von Termessos gelegenen Dorf

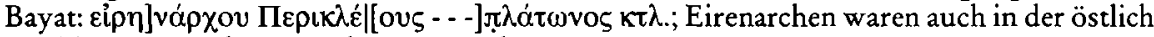
von Termessos gelegenen Ebene tätig, oben S. 2, Anm. 12.

50 Heberdey, TAM (o. Anm. 11) Nr. 88; IGR, III, Nr. 436; $M$. Christol, Un duc dans une inscription de Termessos (Pisidie). Un témoignage sur les troubles intérieurs en Asie Mineure romaine au temps de la crise de l'Empire, in: Chiron 8 (1978) 529-540; AE (1978) 802; SEG XXVIII (1978) 1214; J.-L. Robert, in: BE 9 (1979) Nr. 578; Zimmermann (o. Anm. 20) 266 (Anm. 8) und 273; Mitchell, Native rebellion (o. Anm. 21) 166f.; der im ausgehenden 3. Jahrhundert mit einem speziellen Auftrag (im Kampf gegen die Räuber) als $\delta o v ' \xi$ eingesetzte

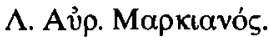

51 Zimmermann (o. Anm. 20) $272 \mathrm{ff}$.

52 E. Petersen, $F$. von Luschan, Reisen im südwestlichen Kleinasien, II: Reisen in Lykien, Milyas und Kibyratis (Wien 1889) 164; Harrison, Nouvelles découvertes (o. Anm. 37) 235ff.; ders., Upland settlements (o. Anm. 37) 110-114.

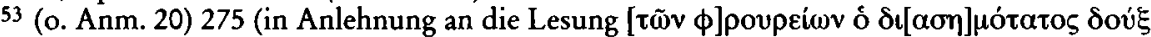

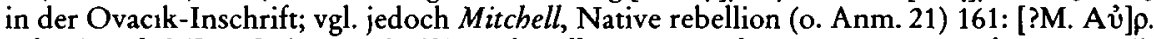

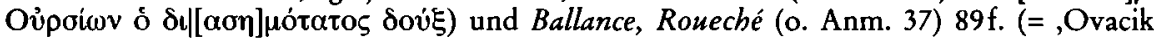
I.A.i'), Z. 1.

54 Schönborn bei Ritter (o. Anm. 22) 809 (Hinweis Fr. Hild). 


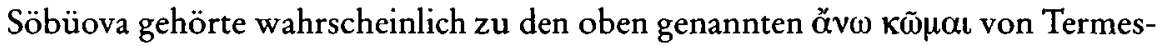
$\operatorname{sos}^{55}$.

Angesichts dieser Umstände ist wohl anzunehmen, daß sich das Territorium von Termessos erheblich weiter als Heberdeys Konstruierung nach dem damals bekannten epigraphischen Material in südwestliche Richtung erstreckte. Freundlicherweise teilte uns Fr. Hild mit, daß die $18 \mathrm{~km}$ nordöstlich von Elmal, $59 \mathrm{~km}$ westlich von Antalya gelegene Gilevgi Kalesi (im zentrallykischen Hochland, zwischen den Dörfern Çobanisa und Gilevgi ${ }^{56}$ ) eine Grenzfestung von Termessos gewesen sein könnte, da sich die termessische Chora so weit nach Südwesten erstreckte ${ }^{57}$.

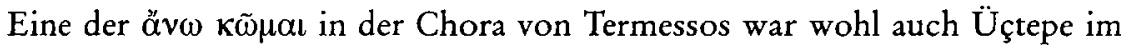
Gebiet des Sinanlı Dağı, $15 \mathrm{~km}$ südwestlich von Termessos ${ }^{58}$, wo sich auf der SüdSeite der Felshöhe Guzkaya(başı) stark verfallene antike Häuser befinden. In der ca. $1 \mathrm{~km}$ südlich gelegenen Flur Beyşik, anscheinend in einem Nekropolenbereich, fanden wir 1995 auf Hinweis Fr. Hilds zwei römische Sarkophage in Arkosolien ${ }^{59}$.

Das nach dem Stadiasmos von Patara ${ }^{60} 101$ Stadien $(19 \mathrm{~km}$ ) von Idebessos (Kozağac1) bzw. 60 Stadien $(11 \mathrm{~km})$ von Lykai ${ }^{61}$ entfernt gelegene, also im mittleren

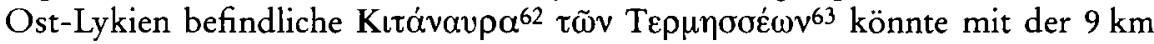
nordöstlich von Idebessos, $40 \mathrm{~km}$ südwestlich von Antalya gelegenen Ruinenstätte von $\mathrm{Sarayc}_{\mathrm{k}}{ }^{64}$, d. h. mit der von Idebessos aus nächsten Ruine einer antiken

55 Zimmermann (o. Anm. 20) 273.

56 Türkei-Karte (o. Anm. 1) IIc/35.

57 Für Gilevgi vgl. Petersen, von Luschan (o. Anm. 52) 165; G. E. Bean, The site of Podalia, in: AnzWien 105 (1968) 161 und Taf. II.4; J. J. Coulton, North Lycia before the Romans, in: J. Borchbardt, G. Dobesch (Hrsg.), Akten des II. Internationalen Lykien-Symposions, Wien 6.-12. Mai 1990, I (Wien 1993) 84; vgl. auch Zimmermann (o. Anm. 20) 273.

58 Türkei-Karte (o. Anm. 1) IIc/38.

${ }^{59}$ Einer von diesen ist unsere Inv.-Nr. T292 mit der Grabstrafe an die Polis (der Termessier?).

$60 \mathrm{Zu}$ diesem für die historische Geographie Lykiens ganz wichtigen Inschriftenfund, der in die Regierungszeit des Claudius datiert und dessen Bearbeitung in den Händen S. Şahins liegt, vgl. S. Sahin, Ein Vorbericht über den Stadiasmus Provinciae Lyciae in Patara, in: Lykia 1 (1994 [1995]) 130-137, Abb. 1-5 = SEG XLIV (1994) 1205.

61 Vermutlich Gödene (Altınyaka), Ağırtaş oder Gedelme; Türkei-Karte (o. Anm. 1) Ile/38.

62 Der vielgestaltig entstellte Name des Ortes kommt im Ortsnamenverzeichnis des Hier.

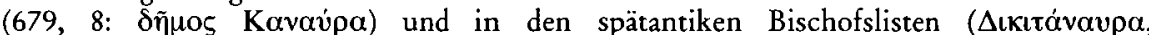

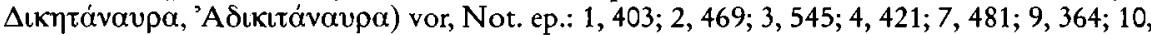

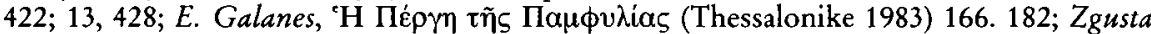
(o. Anm. 23) $222 \$ 425-2$; G. Fedalto, Hierarchia Ecclesiastica Orientalis, I: Patriarchatus Constantinopolitanus (Padova 1988) 248; Nollé (o. Anm. 20) 27 f. W. M. Ramsay, Notes and inscriptions from Asia Minor, in: MdI-A 10 (1885) 343f. hatte nach der hierokleischen Reihenfolge die Ruinenstätte in der Nähe von Kırkgöz lokalisiert, ohne sich jedoch auf feste Beweismittel zu stützen.

63 Stadiasmos von Patara, rechte Breitseite, Z. 37; vgl. F. Isik, H. İ $k$ kan, N. Cevik, Miliarium Lyciae. Patara Yol Kilavuz Anitı. Das Wegweisermonument von Patara. Önrapor/Vorbericht, in: Lykia 4 (1998/1999 [2001]).

64 Türkei-Karte (o. Anm. 1) IId/38; T. A. B. Spratt, E. Forbes, Travels in Lycia, Milyas and the Cibyratis, in company with the late rev. E. T. Daniell (London 1847) I $174 \mathrm{ff}$.; II $203 \mathrm{ff}$; 
Stadt, identisch sein ${ }^{65}$ : Nollé, der Münzen einer bisher unbekannten Münzstätte mit der Legende KITA von einem Schatzfund aus der "Gegend von Antalya “ publizierte, findet es naheliegend, die Stadt Kitanaura mit dem Prägeort dieser auf Grund ihres Stils und der Buchstabenformen in die hellenistische Zeit, ins 2. oder 1. Jahrhundert v. Chr., zu datierenden Münzen zu identifizieren ${ }^{66}$. Es handelt sich also um eine in hellenistischer Zeit selbständige Stadt mit eigener Münzprägung,

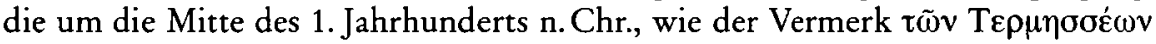
im Meilenmonument von Patara zeigt, unter der Herrschaft von Termessos stand ${ }^{67}$. Wenn die Identifizierung von Saraycik mit Kitanaura richtig ist - bei einer Bereisung konnten wir 1998 im Stadtgebiet keinen epigraphischen Fund machen, der auf den Namen „Kitanaura“ hinweisen könnte -, muß der Südwest-Winkel des termessischen Landbesitzes in römischer Zeit, in der Kitanaura zu Termessos gehörte, also viel weiter nach Süden gerückt werden als von Heberdey angenommen. Die Chora von Termessos reichte dann über das Hochtal von Ovacık tief nach Ost-Lykien hinein bis Kitanaura.

Was den Verlauf der Südwest-Grenze des Territoriums von Termessos zwischen Ovacık und Saraycık betrifft, so könnte es vielleicht bis zum Tal Serkız Alanı im lykischen Hochland, $7 \mathrm{~km}$ südöstlich von Söğle, $19 \mathrm{~km}$ südöstlich von Elmali, $49 \mathrm{~km}$ südwestlich von Termessos, $61 \mathrm{~km}$ westsüdwestlich von Antalya ${ }^{68}$, gereicht haben; hier erhebt sich eine von einer antiken Ringmauer umschlossene Felshöhe an der Nord-Seite des Tales, und von der Felsspitze über den Süd-Hang bis in die Ebene erstreckt sich eine kleine antike Stätte, eine ähnliche Situation wie bei der Hochlandsiedlung bei Ovacık ${ }^{69}$. In diesem Fall würden sich die Territorien von Akarassos und Podaleia nur auf die Elmalı-Ebene beschränken.

Schönborn bei Ritter (o. Anm. 22) 766; Petersen, von Luschan (o. Anm. 52) 150-155; E. Kalinka, TAM, II: Tituli Lyciae linguis Graeca et Latina conscripti, 3: Regiones montanae a valle Xanthi fluminis ad oram orientalem (Wien 1944) 427 (Saracik) Nr. 1222-1230.

${ }^{65}$ Nollé (o. Anm. 20) 16.

${ }^{66}$ Nollé (o. Anm. 20) $12 \mathrm{ff} .16 \mathrm{ff}$.; für weitere, in Saraycık gefundene Münzen mit derselben Legende im Archäologischen Museum Antalya s. M. Arslan, Chr. Lightfoot, C. Tibet, A group of unknown coins in Antalya Museum, in: NCirc 106,1 (1998) $7 \mathrm{f}$.

67 Kitanaura wurde wohl nach den Piratenkriegen, in denen es vermutlich auf Seiten des Zeniketes stand, von den Römern dem termessischen Herrschaftsbereich zugewiesen, vgl. dazu die Überlegungen von Nollé (o. Anm. 20) 22-25, der als terminus ante quem für die Prägung der Münzen an das Jahr 74 v. Chr. denkt; vgl. auch ders., Münzen als Zeugnisse der Hellenisierung Kleinasiens, in: U. Peter (Hrsg.), Stephanos nomismatikos. Edith Schönert-Geiss zum 65. Geburtstag (Berlin 1998) $507 \mathrm{f}$. Die in hellenistischer Zeit selbständige Polis und spätere Kome Kitanaura in der Chora von Termessos wurde also vielleicht erst im 6. Jahrhundert Bistum, das als Dikitanaura in den Notitiae episcopatuum erscheint. Für das Ethnikon Kıtıatí 5 , das wohl die Herkunft einer Person aus Kita(-Naura) bezeichnet, s. eine Grabstele aus Patara, S. Şabin, Epigraphische Mitteilungen aus Antalya I: Inschriften aus Pamphylien und Lykien, in: EA 31 (1999) 49, Nr. 15, Z. 4.

68 Auch Serkız Ovası bzw. Serkız/Sirkis Yaylası; vgl. Türkei-Karte (o. Anm. 1) IId/35-36. ${ }^{69}$ Für Serkız Alanı vgl. Petersen, von Luschan (o. Anm. 52) 163; G. E. Bean, Journeys in Northern Lycia 1965-1967 (Wien 1971) 33; R. M. Harrison, Survey in central Lycia, in: AST 1 (1983 [1984]) 76 mit Fig. 6 f. auf S. 245 und H. Hellenkemper, Fr. Hild, TIB, VIII: Lykien und Pamphylien, s.v. „Serkız Alanı" (in Vorbereitung). 
Im Forschungsjahr 1999 haben wir in der Flur Karabucak, etwa 1,5 km westlich von Yarbaşı̧̧andırı in Nordost-Lykien, am westlichen Ufer des Çandır Çayy ${ }^{70}$, die Grabinschrift einer Priesterin aus $\mathbf{M} v \alpha \rho \alpha^{71}$ gefunden, das offenbar mit Phaselis in Sympolitie stand und dessen Territorium ${ }^{72}$ an die Chora von Termessos im Süden angrenzte. Nach dem Stadiasmos von Patara war die Mvapıri eine Landschaft in

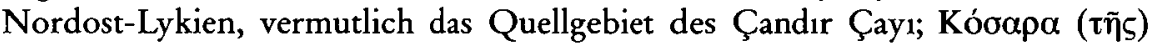

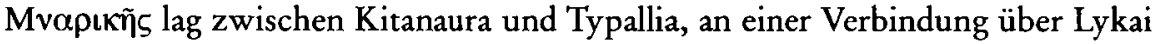
nach Kitanaura und als Ausgangspunkt einer Straße nach Phaselis.

Mitchell bezweifelte, daß der Fundort der bereits genannten Ehrung für Trokondas in Bayat selbst die Stelle der ansonsten unbekannten Stadt Typallia war, und schlug für die Lokalisierung die Ruinen einer kleinen, namenlosen pisidischen Stadt gegenüber von Sandalion in östlicher Richtung auf den höheren Hängen des Davras Dağ1 vor ${ }^{73}$. Dieser Vorschlag, der zunächst plausibel erscheint, wird allerdings durch den Stadiasmos von Patara zweifelhaft, in dem ein Tขлó $\lambda \lambda \iota \alpha$ an einem Weg vom bereits genannten Kitanaura über Kosara ${ }^{74}$ ( $\tau \tilde{\eta} \zeta$ Mv $\alpha \rho \kappa \tilde{\eta} \varsigma$ ) nach Trebenna (Trabenna) ${ }^{75}$ in Ost-Lykien, zwischen Kosara und Trebenna genannt wird ${ }^{76}$. Aus diesem topographischen Grund haben wir im Jahre 1999 in der im 19. Jahrhundert ohne historische und archäologische Anhaltspunkte versuchsweise mit Marmara ${ }^{77}$ gleichgesetzten Ruinenstätte in den West-Hängen über dem Çandır-Tal in Nordost-Lykien, heute Karabel ${ }^{78}$, inten-

$7031 \mathrm{~km}$ südwestlich von Antalya; Türkei-Karte (o. Anm. 1) IIç/38.

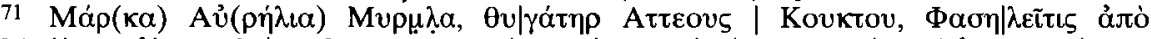

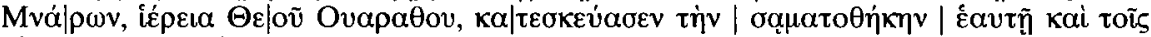

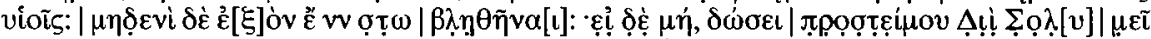

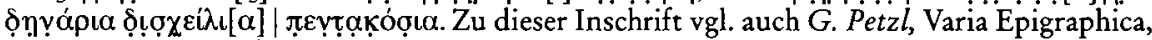
in: EA 33 (2001) $51 \mathrm{f}$.

72 Stadiasmos von Patara, rechte Breitseite, Z. 38f., 47; vgl. Işık, İskan, Çevik (o. Anm. 63). Die Stelle von Mnara ist höchstwahrscheinlich die etwa $14 \mathrm{~km}$ sö. des Fundortes [1300$1400 \mathrm{~m}$ hoch] gelegene, antike Siedlung (Marmara) auf Kavak Dağı in Türkei-Karte (o. Anm. 1) IId/39, die zumindest in hellenistischer Zeit den Status einer Polis innehatte und in römischer Zeit im Territorium von Phaselis aufging; vgl. S. Şabin, Epigraphische Mitteilungen aus Antalya V: Olbia und einige andere Küstenorte bei Kemer in Westpamphylien, in: EA 33 (2001) Karte 1 nach S. 156; B. İplikçioğlu, Doğu ve Kuzeydoğu Lykia-Güneybatı, Pisidia Epigrafik-Tarihi Coğrafi Yüzey Araştırmaları Projesi 2000 Y1lı Çalışmaları, in: AST 19, 2 (2001 [2002]) 130, Anm. 8; demnächst auch Hellenkemper-Hild (o. Anm. 69), s.v. „Mnara“" ${ }^{73}$ Mitchell, Amyntas in Pisidien (o. Anm. 21) 100.

74 Vielleicht Kozarası (Türkei-Karte [o. Anm. 1] IId/38), $5 \mathrm{~km}$ nordöstlich von Saraycık; s. Spratt, Forbes (o. Anm. 64) I 174, 179; Ritter (o. Anm. 22) 1172 f.

$751 \mathrm{~km}$ südöstlich von Geyikbayırı Köyü bzw. 1,5 km nordöstlich von Karcıbayırı Mahallesi (Çağlarca), $17 \mathrm{~km}$ westlich von Antalya (Türkei-Karte [o. Anm. 1] IIc/39); vgl. S. Eyice, Trebenna: Antalya Yakınında Eski Bir Şehir Kalıntısı, in: Türkiye Turing ve Otomobil Kurumu Belleteni 58/337 (1977); Zgusta (o. Anm. 23) 633 \$1363-2.

76 Rechte Breitseite, Z. $38 \mathrm{ff}$; vgl. Işık, İskan, Çevik (o. Anm. 63).

$77 \mathrm{Vgl}$. Heberdey (o. Anm. 4) 5, Fig. 1.

78 Etwa $1 \mathrm{~km}$ westnordwestlich von Çitdibi Köyü, $27 \mathrm{~km}$ südwestlich von Antalya (TürkeiKarte [o. Anm. 1] IIç/39); vgl. Spratt, Forbes (o. Anm. 64) I 205-207, II 12; Schönborn bei Ritter (o. Anm. 22) 635; Petersen, von Luschan (o. Anm. 52) 148f., Inschriften Nr. 177-180; 
sive Forschungen durchgeführt und konnten dabei auf Grund eines Neufundes ${ }^{79}$ und eines Corrigendum ${ }^{80}$ (Pace) feststellen, daß diese antike Siedlung mit dem in der Bayat-Inschrift und im Stadiasmos von Patara genannten Tvлá $\lambda \lambda \iota \alpha$ identisch ist $^{81}$ und daß dieses Typallia mit Termessos in Sympolitie stand. Während derselben Forschungen wurde in der Flur Karagedeller, etwa 1-1,5 km ostsüdöstlich von Çitdibi Köyü, also sehr wahrscheinlich im Territorium von Typallia, ein Sarkophag gefunden, in dessen Inschrift ${ }^{82}$ ein Termessier genannt wird, allerdings wohl als Steinmetz ${ }^{83}$. Auf Grund der Sympolitie der Typallioten mit den Termessiern war der bereits genannte Krieg gegen Sandalion offenbar eine gemeinsame Sache zwischen Termessos und Typallia, so daß die Typallioten die Bestattung eines erfolgreichen Kommandeurs dieses Krieges aus Termessos in seinem Landbesitz mit einem Ehrendekret gefeiert haben, wobei anzunehmen ist, daß dort noch andere Ehrendenkmäler für Trokondas (auch seitens Termessos) gesetzt worden sind.

In TAM III,1, Nr. 895 und 897 führt die im Bergland südwestlich von Termes-

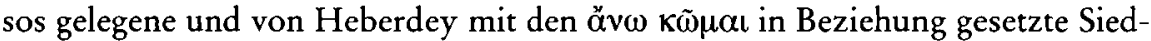
lung keinen Eigennamen, sondern es erscheint statt dessen die Bezeichnung tò $\pi \varepsilon p ı$ ó̀ıov, die Romanelli ${ }^{84}$ im Sinne von „Zitadelle“ verstanden hatte ${ }^{85}$. Nach

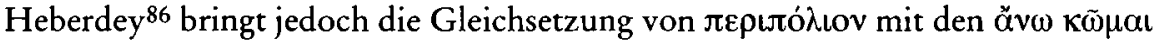
die richtige Erklärung: Wie $\pi \varepsilon \rho i \chi \omega \rho \circ \zeta$ allgemein das die Stadt umgebende Terri-

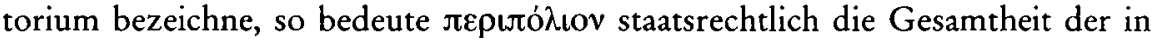
diesem Bezirk gelegenen Gemeinden, die sich unter diesem Namen zu einer halbselbständigen Gemeinschaft vereinigt hätten.

Wir dürfen aus den bisher bekannt gewordenen Zeugnissen für $\pi \varepsilon \rho ı$ ó̀ı $\alpha$ ent-

Pace (o. Anm. 19) $60 \mathrm{ff}$; C. Anti, Esplorazioni archeologiche nella Licia e nella Pamfilia, in: MonAL 29 (1923) $661 \mathrm{f}$. (Karte); V. Viale, Relazione sull'attività della missione archeologica di Adalia nell'anno 1922, in: ASAA 8/9 (1925/1926 [1929]) 383 f.; Fr. Stark, Alexander's path from Caria to Cilicia (London 1958) 250.

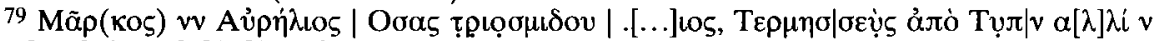
$\omega[v, \kappa] \alpha[\tau \varepsilon \sigma \kappa] \varepsilon[\dot{v} \alpha] \mid \sigma \varepsilon v \varepsilon \varepsilon \alpha v \tilde{\varphi} \kappa \kappa \lambda$.

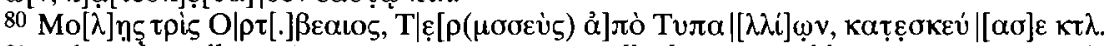

81 Vgl. auch Nollé (o. Anm. 20) 16, Anm. 11; Hellenkemper, Hild (o. Anm. 69) s.v. „Typallia“.

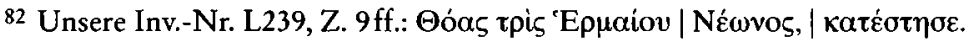

${ }^{83}$ Die Gegend gegenüber von Typallia, also die Ost-Seite des Çandır Çayı, gehörte zur Chora von Phaselis, vgl. die Sarkophag-Inschrift aus der Flur Zindan Taşı bzw. Zindan Yakası westlich von Akçaisa (Türkei-Karte [o. Anm. 1] IIç/39) mit der Grabstrafe an die Polis der Phaseliten, Anti (o. Anm. 78) 735-738, Nr. 3 = SEG VI (1932) 735.

84 Paribeni, Romanelli (o. Anm. 5) 198.

85 Dies begründete er damit, daß die unbedeutende Siedlung, statt den eigenen Namen zu nennen, sich als Befestigung der großen Nachbarstadt Termessos bezeichnet hätte, um sich selbst zu rühmen. Dieser Vorschlag wurde jedoch von Heberdey abgelehnt, da diese Bedeutung des Wortes nicht zu belegen, auch etymologisch schwer zu rechtfertigen sei und weil solch eine Rolle der Siedlung gegenüber Termessos gar nicht zu den tatsächlichen Verhältnissen passe, Heberdey (o. Anm. 4) 6.

86 (o. Anm. 4) 6f.; Heberdey, TAM (o. Anm. 11) 271. 


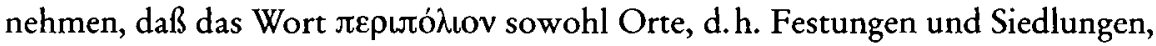
als auch Gemeinschaften verschiedener Natur bezeichnete ${ }^{87}$ :

Der früheste Beleg für ein Peripolion in Kleinasien ist die Fluchstele von Teos um 470 v. Chr. ${ }^{88}$, vielleicht im Sinne von "Festung "89. Ein im lykischen Xanthos gefundener und in die zweite Hälfte des 2. Jahrhunderts v. Chr. gehörender Iso-

87 Das Wort $\pi \varepsilon \rho$ เó $\lambda$ เov ist von W. Dittenberger im Kommentar zur SIG 3 , 570 als Ableitung von $\pi \varepsilon p i \tau \tau$ tos erklärt worden. Für die epigraphisch belegten Beispiele aus Halasarna, Kamiros und Potidaion, die in die Zeit zwischen ca. 225 bis ca. 153 v. Chr. datieren, vgl. F. G. Maier, Griechische Mauerbauinschriften II: Untersuchungen (Heidelberg 1961) 78ff.; vgl. auch die Zeugnisse ders., Griechische Mauerbauinschriften I: Texte und Kommentare (Heidelberg 1959) $174 \mathrm{ff} .$, Nr. 46 (aus Halasarna auf Kos); 183 ff., Nr. 49 (Kamiros auf Rhodos) und $188 \mathrm{ff}$., Nr. 50 (Potidaion auf Karpathos); zu den zahlreichen anderen griechischen Begriffen für Festungsanlagen auf der Chora vgl. L. Robert, F. G. Maier, Griechische Mauerbauinschriften, in: Gnomon 42 (1970) $588 \mathrm{f}$. und 598ff.; zur Situation auf Kos vgl. auch $P$. Baker, Cos et Calymna, 205-200 a.C.: Esprit civique et défense nationale (Québec 1991) 38-47;

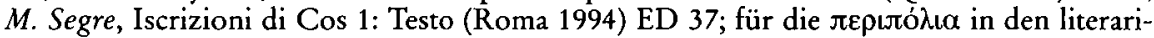
schen Nachrichten aus Attika Thuk. 3, 99; 6, 45; 7, 48; vgl. auch Dion. Hal., ant. 9, 56; Maier, II: Untersuchungen (o.) 79f. mit Anm. 43 und M. Zimmermann, Untersuchungen zur historischen Landeskunde Zentrallykiens (Bonn 1992) 80, Anm. 110. Diese sind wohl zum Schutz der Landbevölkerung angelegte, aber auch zur Verteidigung des Herrschaftsbereiches dienende Festungen (vgl. auch Zimmermann [o.] 80), wobei die Bezeichnung in den Inschriften für eine befestigte Gemeinde, d.h. eine größere Anlage von burgartigem Charakter, bei den

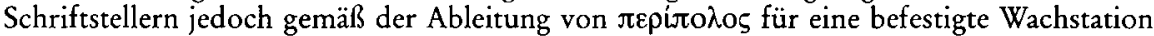
gilt (vgl. Maier, II: Untersuchungen [o.] $79 \mathrm{f}$. mit den Anm. $43 \mathrm{ff}$. Da in den oben genannten Inschriften aus Halasarna, Kamiros und Potidaion von einem „unbefestigten Peripolion“ die Rede ist, bezeichnet das Wort nach Maier nicht - wie etwa фpoúplov - „in rein technischem Sinn die Festung als Bauwerk“, sondern den „festen Platz“ einer ländlichen Gemeinde der Polis [deren Befestigung erst bei Gefahr eines Angriffs durch fremde Mächte erneuert wird], und der Begriff könne zwar die eigentliche Verfassungsbezeichnung der Orte nicht ersetzen, aber doch stellvertretend dafür gebraucht werden; vornehmlich auf Grund der Zeugnisse aus Termessos, Heberdey, TAM [o. Anm. 11] Nr. 895 und 897, sieht Maier in dem Wort лєрıлó $\lambda$ เov „eine eindeutig die politische Gemeinde bezeichnende Sonderbedeutung“, zu der das Mitklingen staatsrechtlicher Begriffe geführt habe, und der Sinn als „Befestigung einer gröBeren Gemeinde" sei eine Eigentümlichkeit der rhodischen Welt des 3. und 2. Jahrhunderts v. Chr. [vgl. auch Zimmermann (o.) 81; Schuler (o. Anm. 7) 49]. Zur Verteidigung des Territoriums in den pontischen griechischen Städten der hellenistischen und römischen Zeit vgl. $A$. Avram, G. Vlad Nistor, Apàrarea teritoriului în cetàtile Greceşti şi problemele zonei Pontice, in: SCIV 33 [1982] 365-376, wo Befestigungen in zwei Kategorien, d.h. als Festungen

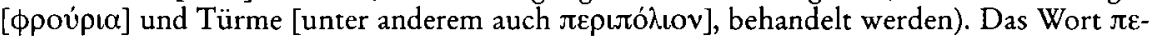

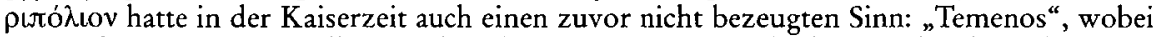
eine Befestigung keine Rolle gespielt zu haben scheint. In Inschriften aus dem karischen Stratonikeia ( $M$. Ç. Şabin, IK 21: Die Inschriften von Stratonikeia, Teil I: Panamara [Bonn 1981] Nr. 224; ders., IK 22,1: Die Inschriften von Stratonikeia, Teil II,1: Lagina, Stratonikeia und Umgebung [Bonn 1982] Nr. 524. 529.663.664. 673.678. 682. 685.699. 701. 704. 1101) ist mit

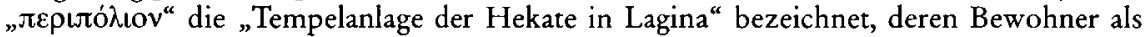

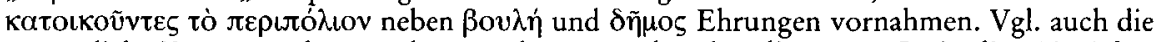
vorzügliche Zusammenfassung des Forschungsstandes über die Frage „Peripolion“ in Schuler (o. Anm. 7) 45-49.

$88 \mathrm{SIG}^{3}, 38, \mathrm{Z} .15$.

89 Vgl. Maier, II: Untersuchungen (o. Anm. 87) 79, Anm. 43; Schuler (o. Anm. 7 ) 46 mit Anm. 172. 
politievertrag zwischen Xanthos und $\mathrm{Myra}^{90}$ zeigt, daß die $\pi \varepsilon \rho \iota \pi \dot{\lambda} \lambda \iota \alpha$ von den jeweiligen $\pi \hat{\lambda} \lambda \varepsilon \iota \varsigma$ abgesondert, jedoch von ihnen abhängig waren und somit einen Bestandteil dieser ausmachten, da nach dem Wortlaut der Klausel die Angehöri-

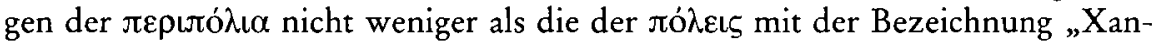
thier" bzw. „Myräer" erscheinen ${ }^{91}$. Nach Wörrle ${ }^{92}$ waren diese Peripolia rechtlich nichts anderes als abhängige $\delta \tilde{\eta} \mu \circ \mathrm{im}$ Polisgebiet ${ }^{93}$. Südsüdwestlich von Bağlıca ${ }^{94}$ in Zentrallykien entdeckte Davies die Ruinen antiker Siedlungen mit Befestigungsanlagen ${ }^{95}$. In der in die Zeit des 2.-1. Jahrhunderts v. Chr. zu datierenden Inschriff ${ }^{96}$ auf einem Sarkophag einer der zwischen diesen Anlagen befindlichen

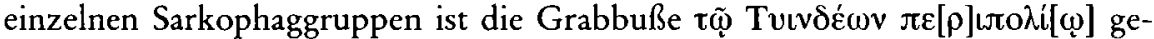

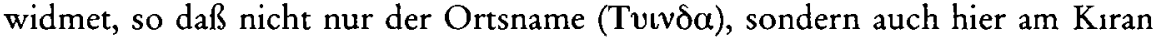
Dağ 1 zwischen den Territorien von Phellos und Kyaneai die Existenz eines $\pi \varepsilon p \iota \pi$ ó̀ı $\mathbf{v}$ festgestellt werden konnte ${ }^{97}$, das offensichtlich, vergleichbar mit dem

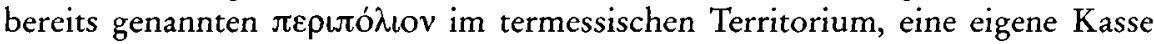
und demnach eine (begrenzt) eigenständige Verwaltung bzw. lokale Autonomie hatte ${ }^{98}$. Ein Peripolion ist auch in Asarönü bei Limyra bezeugt ${ }^{99}$ : Hier wird in

90 J. Bousquet, P. Gauthier, Inscriptions du Létôon de Xanthos, in: REG 107 (1994) 319-347 = SEG XLIV (1994) 1218; vgl. auch Schuler (o. Anm. 7) $46 \mathrm{f}$.

91 Bousquet, Gauthier (o. Anm. 90) 339.

92 Epigraphische Forschungen zur Geschichte Lykiens VII: Asarönü, ein Peripolion von Limyra, in: Chiron 29 (1999) 361.

${ }^{93}$ Für Zugehörigkeit zu Bürgerschaftsgliederungen in Lykien vgl. ebd. $359 \mathrm{f}$.

${ }_{94}$ Nordöstlich von Isinda, ca. $10 \mathrm{~km}$ ostnordöstlich von Antiphellos; Türkei-Karte 1:200000, Harita Genel Müdürlüğü, İ IV: Kaş (Ankara 1944 [1950]) IIh/32.

95 G. Davies, Greek inscriptions from Lycia, in: JHS 15 (1895) 109.

96 Davies (o. Anm. 95) 109, Nr. 19, Z. 3 f.; zur Datierung vgl. Zimmermann (o. Anm. 87) 80 mit Anm. 108.

97 In einer nahe der obigen Inschrift gefundenen, aber noch nicht publizierten Grabinschrift (auch aus hellenistischer Zeit) sei der Empfänger der Grabstrafe der Demos von Kyaneai, so daß die Zugehörigkeit dieses westlichen Hügellandes zum Territorium von Kyaneai bewiesen werden könne; wenige hundert Meter weiter südwestlich beginne zu dieser Zeit das Gebiet von Phellos, Zimmermann (o. Anm. 87) 81; vgl. auch Fr. Kolb, M. Zimmermann, Forschungsziel, Forschungsstand und historische Einleitung, in: Kyaneai 1989: Vorbericht über Feldforschungen im Yavu-Bergland bei Kaş, in: MdI-I 41 (1991) 198. Für Tyinda vgl. W. Ruge, in: RE VII A,2 (1948) 2557, s.v. „Tyindeis“; L. Robert, Villes d'Asie Mineure: Etudes de géographie ancienne (Paris 1935, 21962) 142f.; G. E. Bean, Lycian Turkey: An archaeological guide (London 1978) 105; Zgusta (o. Anm. 23) 639 \$1380; Zimmermann (o. Anm. 88) 61-67, 77-83, $99 \mathrm{f}$. (bisher die eingehendste Behandlung des Gebietes); Fr. Kolb, Stadt und Land im antiken Kleinasien: Der Testfall Kyaneai, in: J. H. M. Strubbe, R. A. Tybout, H. S. Versnel (eds.), ENERGEIA: Studies on ancient history and epigraphy presented to $H$. W. Pleket (Amsterdam 1996) 105; Schuler (o. Anm. 7) 47f.; zur Lage s. zuletzt Fr. Kolb, 1997 Yil Kyaneai Yüzey Araştırması, in: AST 16,2 (1998 [1999]) 391 und Wörrle (o. Anm. 92) $358 \mathrm{f}$. 98 Zimmermann (o. Anm. 87) 79f.; ders. (o. Anm. 20) 273; Kolb, Stadt und Land (o. Anm. 97) 105.

99 Türkei-Karte (o. Anm. 94) IIg/35-36; G. Stanzl, in: J. Borchhardt, Grabungen und Forschungen in Limyra aus den Jahren 1984-1990, in: JÖAI 61 (1991/1992) Beibl. 127 (Karte); Th. Marksteiner, Die befestigte Siedlung von Limyra: Studien zur vorrömischen Wehrarchitektur und Siedlungsentwicklung in Lykien unter besonderer Berücksichtigung der 
einer nicht sehr lange nach der Constitutio Antoniniana gesetzten Inschrift 100

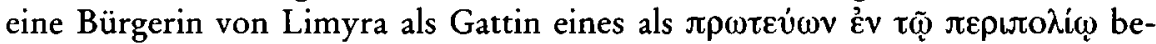
zeichneten Mannes erwähnt, der als iєpєù $\tau_{\tilde{\omega}} v \Sigma \varepsilon \beta \alpha \sigma \tau \tilde{\omega} v$ mit seiner Gemahlin den ersten Rang in der Hierarchie der städtischen Amts- und Würdenträger erlangt hatte ${ }^{101}$. Gemeinsam ist vor allem den beiden lykischen Peripolia von Tyinda und Asarönü, daß sie politisch zu einer Polis gehörten und an der Grenze der Chora lagen ${ }^{102}$.

Einige in den Forschungsjahren 1996 und 1997 in zwei Ruinenstätten in der termessischen Chora von uns gefundenen und in die römische Kaiserzeit gehörenden Inschriften haben auch zu dem Thema „Peripolion“ beigetragen ${ }^{103}$ : In der von Heberdey mit den ởv $\kappa \tilde{\omega} \mu \alpha$ in Beziehung gesetzten Ruinenstätte wurde

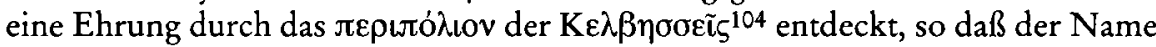
dieses antik-frühbyzantinischen Siedlungsplatzes nunmehr mit Sicherheit als

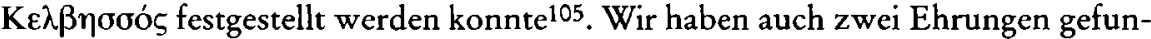

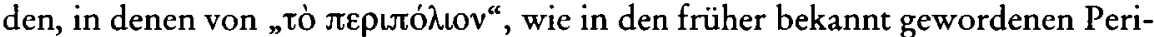

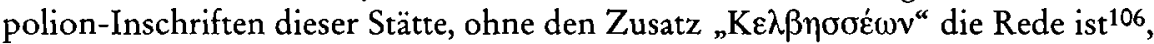
und dieses $\pi \varepsilon \rho \iota \pi$ ó der Stätte nur als Empfänger des Strafgeldes im Falle unbefugter Benützung des Grabes. Die andere Ruinenstätte ist der ca. $8 \mathrm{~km}$ südöstlich von Termessos, nordwestlich oberhalb von Doyran und ca. $16 \mathrm{~km}$ westnordwestlich von Antalya gelegene antik-frühbyzantinische Siedlungsplatz auf einem Ausläufer des Göldağ ${ }^{107}$, der heute „Keldağ“ genannt wird: Auf Grund einer hier entdeckten Ehrung, dies-

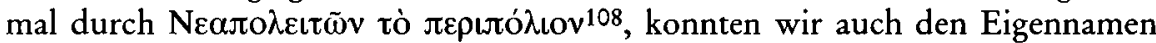
dieser Siedlung mit Sicherheit ermitteln ${ }^{109}$. Dieses $\pi \varepsilon \rho$ เлó $\lambda\llcorner$ เov erscheint ebenso in

klassischen Periode (Wien 1997) 220, Anm. 69; A. Konecny, Hellenistische Turmgehöfte in Zentral- und Ostlykien (Wien 1997) 95, Anm. 63 und zuletzt Wörrle (o. Anm. 92) 353-365 mit Abb. 1-5 auf S. 366-370.

100 Wörle (o. Anm. 92) $353 \mathrm{f}$.

101 Vgl. Wörrle (o. Anm. 92) 355.

102 Vgl. auch Wörrle (o. Anm. 92) 359.

$103 \mathrm{Vgl}$. auch Íplikçioğlu (o. Anm. 20) $312 \mathrm{f}$.

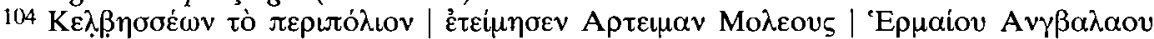

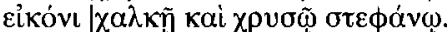

105 Vgl. auch B. İplikçioğlu, G. Çelgin, V. Çelgin, Termessos ve Egemenlik Alanı EpigrafikTarihi Coğrafi Yüzey Araştırmaları (1992-1996 Çalışmaları), in: AST 15,1 (1997 [1998]) $378 \mathrm{ff}$. Der Empfänger der Strafsumme in drei am selben Ort neugefundenen Grabinschriften

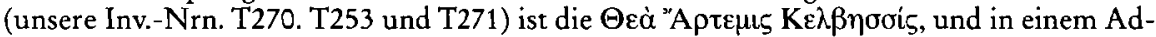
dendum-Corrigendum zu Heberdey, TAM (o. Anm. 11) (unsere Inv.-Nr. T280) gemeinsam

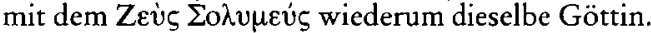

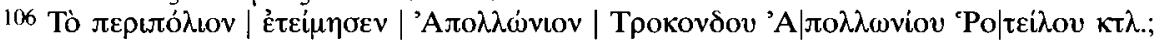

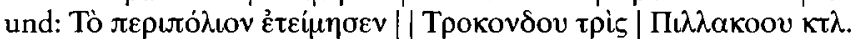

107 Türkei-Karte (o. Anm. 1) IIb/39.

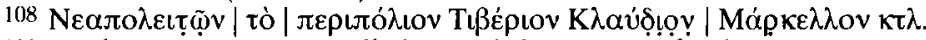

109 Auch in unserer im nördlichen Teil der Stätte gefundenen Inv.-Nr. T321 ist der Graberrichter als oik 
einer Inschrift ${ }^{110}$ von einer byzantinischen Kirche im südlichen Teil des Ortes als Vollender des Tempels des Zzì $\Sigma \omega t \eta \dot{\rho} \rho^{111}$.

Die in der Stadt selbst gefundene Inschrift TAM III,1, Nr. 1 ist ein weiteres

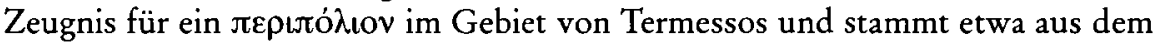
2. Jahrhundert v. Chr. In Z. 12 dieses schlecht erhaltenen Ehrendekretes ist nun, wie auf dem Sarkophag von Tyinda und in anderen Peripolion-Inschriften von

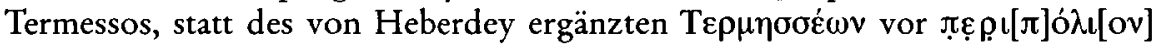

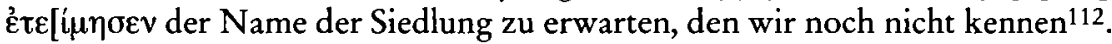

Peripolion erscheint in den bereits angeführten Grabinschriften aus dem lykischen Tyinda und aus Kelbessos - wie in den sonstigen ähnlichen Klauseln ${ }^{113}$ dort, wo die Namen der jeweiligen Gemeinden als Empfänger des Strafgeldes genannt werden, oder, wie die termessischen ${ }^{114}$ und die karischen ${ }^{115}$ Beispiele zeigen, als lokale politische Organisation ${ }^{116}$ bzw. als Zentrum einer Lokalgemeinde ${ }^{117}$, die selbständig Ehrungen beschließen durfte. Die $\pi \varepsilon \rho \iota \pi o ́ \lambda \iota \alpha$ von Tyinda, Kelbessos und Neapolis machen sich überdies gelegentlich auch mit dem Namen der jeweiligen Gemeinde kenntlich. Demnach bezeichnete dieses Wort nicht wie in der Deutung Heberdeys die „staatsrechtliche Gesamtheit der in einem Bezirk gelegenen Gemeinden" ${ }^{118}$, sondern eine Siedlung im Inneren oder an der Grenze eines Polisterritoriums ${ }^{119}$ und zugleich eine Gemeinschaft mit

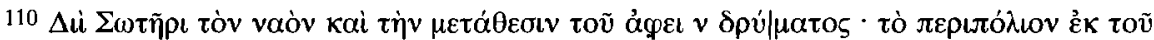

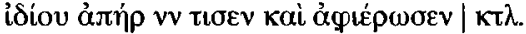

111 Die Zugehörigkeit dieser Stätte zur Chora von Termessos wird auch durch unsere Inv.-

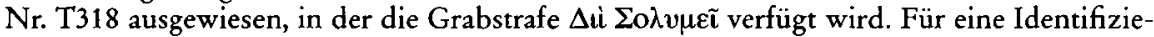
rung mit dem spätantiken Iobia vgl. Hellenkemper, Hild (o. Anm. 69) s.v. „Iobia“. Iobia wurde wie Eudokias (s. oben Anm. 11) im 5. Jahrhundert Polis und Bistum der spätantiken Provinz Pamphylia (Hier. 680,1); Sabinianos nahm an der Synode von Konstantinopel im Jahre 448 als Bischof von Termessos, Eudokias und Iobia teil (ACO, II 1, 1 S. 146, Nr. 18; II 2, 1 S. 20; II 3, 1 S. 129, Nr. 18); während Iobia nur für das 5. Jahrhundert bezeugt ist, erscheinen Termessos und Eudokias bis in das 12. Jahrhundert als Bistümer in den Notitiae episcopatuum; für Iobia vgl. auch W. Ruge, in: RE IX,2 (1916) 1838, s.v. „Iobia“.

112 Vgl. auch Schuler (o. Anm. 7) 48, Anm. 183 und Wörrle (o. Anm. 92) 364.

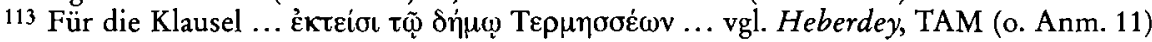
Nr. 220. 331. 382. 383. 508. 525. 532.607. 646. 650. 665. 699. 831.

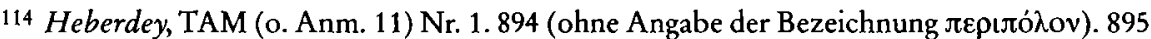
und 897 sowie unsere Inv.-Nrn. T254. T255. T260 und T312.

115 Vgl. oben Anm. 88.

116 In Termessos mit einem åp $\chi \omega v$ an der Spitze; vgl. Heberdey, TAM (o. Anm. 11) Nr. 893. 898; Heberdey (o. Anm. 4) 6; ders. (o. Anm. 1) 766f.; Zimmermann (o. Anm. 20) 272f.; Schuler (o. Anm. 7) 48 mit Anm. 185.

117 Vgl. Bousquet, Gauthier (o. Anm. 91) 340; Schuler (o. Anm. 7) 49 mit Anm. 188; zu den einschlägigen Strabon-Stellen $(7,7,6 ; 14,2,22 ; 16.2,10 ; 17,3,21)$ vgl. Wörrle (o. Anm. 92)

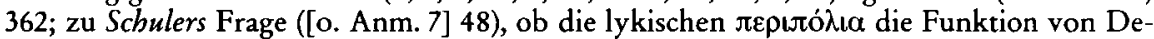
menzentren hatten, vgl. Wörrle (o. Anm. 92) Anm. 36.

118 Vgl. auch Maier, II: Untersuchungen (o. Anm. 87) 80, Anm. 45; Bousquet, Gautbier (o. Anm. 90) 340, Anm. 82 und Schuler (o. Anm. 7) 48, Anm. 183.

119 Vgl. auch Bousquet, Gautbier (o. Anm. 90) 340. Während leicht zu ermitteln ist, daß die Peripolia von Termessos, Kyaneai und Limyra ganz nahe der Polisgrenze lagen, scheinen dagegen diejenigen von Xanthos und Myra als Bestandteile der jeweiligen Jó $\lambda \varepsilon ı \zeta$ im ganzen 
(begrenzt) eigenständiger Verwaltung ${ }^{120}$ und eigener öffentlicher Kasse bzw. ein administratives Zentrum für die ländliche Bevölkerung, eine Verwaltungseinheit der Chora. Einige $\pi \varepsilon \rho \iota \pi o ́ \lambda \iota \alpha$ waren, wie die Beispiele von Tyinda, Kelbessos und Neapolis zeigen, ländliche Siedlungen an der Polisgrenze: $\mathrm{Daß}$ Tyinda zwischen den Territorien von Phellos und Kyaneai nahe der Grenzlinie liegt, spricht nach Zimmermann ${ }^{121}$ dafür, daß̧ die Siedlung aus einer Grenzfestung hervorgegangen sein könnte ${ }^{122}$. Die Peripolia von Tyinda und Asarönü sind allerdings unbefestigte, wohl nicht auf Grund des Grenzverteidigunsbedürfnisses entstandene Kleinpoleis ${ }^{123}$. Mit Wörrle ${ }^{124}$ sind wir der Meinung, daß die lykischen und die termessischen Peripolia ehemals selbständige Orte mit eigenem Hinterland und deshalb nach der Integration in die Chora einer Polis abhängige $\delta \tilde{\eta} \mu \iota^{125}$ mit besonderem Rang und begrenzter Selbstverwaltung waren. Daher braucht die Lage von Kelbessos und Neapolis jeweils an der südlichen Polisgrenze von Termessos zu Trebenna nicht unbedingt mit einstigem fortifikatorischem bzw. Grenzverteidigungszweck erklärt zu werden ${ }^{126}$, obwohl in Kelbessos Spuren einer Befestigung in Richtung O, d.h. auf der Nekropolenseite, sichtbar sind. Solche Spuren sind zwar auf der westlichen Seite nicht zu sehen, doch befindet sich dieser Rand der

Territorium verstreut gewesen zu sein, bzw. die Lage dieser Peripolia im Territorium bleibt unbekannt (zur Diskussion über die Peripolia angesichts der Organisation des Territoriums im hellenistischen Zeitalter in Lykien ["Perioikoi“ und "Sympolitien“] s. dies. a.O.: $341 \mathrm{ff}$.; vgl. dazu auch Schuler [o. Anm. 7] 47 mit Anm. 176 und Wörrle [o. Anm. 92] 362f. mit Anm. 37 und 38).

120 Über die Organisation der Verwaltung der termessischen Peripolia, an deren Spitze ein åp $\chi \omega v$ stand (vgl. oben Anm. 116), kann im einzelnen nichts gesagt werden; zur Verwaltung der attischen vgl. Hommel, in: RE XIX (1937) 852 ff., s.v. „Peripoloi“ und J. H. Kroll, F. W. Mitchell, Clay tokens stamped with the names of Athenian military commanders, in: Hesperia 49 (1980) 86-89; zur militärischen Organisation der Phrouria auf dem Gebiet des lykischen Xanthos: J.-L. Robert, Fouilles d'Amyzon en Carie, 1: Exploration, histoire, monnaies et inscriptions (Paris 1983) $125 \mathrm{ff}$;; zu den Amtsträgern in den Peripolia vgl. auch $L$. Robert, Hellenica: Recueil d'épigraphie, de numismatique et d'antiquités grecques X (Paris 1955) $283 \mathrm{ff}$. und J.-L. Robert, in: BE (1973) $110 \mathrm{f}$.

121 (o. Anm. 87) 80-83.

122 Vgl. auch Kolb, Zimmermann (o. Anm. 97) 198.

123 Vgl. auch Scbuler (o. Anm. 7) 48 mit Anm. 181; Wörrle (o. Anm. 92) $361 \mathrm{f}$.

124 (o. Anm. 92) 361 f.; vgl. auch Schuler (o. Anm. 7) 49.

125 Auch die $\pi \varepsilon \rho \iota \pi o ́ \lambda \iota \alpha$ von Halasarna auf Kos, Potidaion auf Karpathos und Kamiros (vgl. oben Anm. 87) waren in Abhängigkeit geratene Kleinpoleis.

${ }_{126}$ Vgl. Zimmermann (o. Anm. 87) 81, wo er einen Zusammenhang zwischen fortifikatorischer Funktion und lokaler Selbstverwaltung der Peripolia herstellt. Immerhin scheint ein fortifikatorischer Aspekt mit dem Siedlungsbegriff Peripolion verbunden zu sein. Zu den

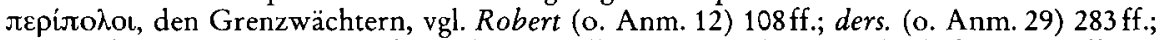
J.-L. Robert, in: BE (1973) 110 f. Der für das hellenistische Telmessos überlieferte Begriff von "(h)orophylakia“ gehört auch in den Zusammenhang der Grenzbefestigung, vgl. dazu $M$. Wörrle, Epigraphische Forschungen zur Geschichte Lykiens III, in: Chiron 9 (1979) $96 \mathrm{ff} . ;$ J.-L. Robert, La Carie: Histoire et géographie historique, avec le recueil des inscriptions antiques. La plateau de Tabai et ses environs, II (Paris 1954) 42. 282; dies. (o. Anm. 120) $101 \mathrm{ff}$. (Betonung der Funktion als „orophylakia“, als „gardes des montagnes“ im Gegensatz zur älteren Interpretation als Grenzwächter). 
Stätte naturbefestigt an einem Steilhang. Letzteres gilt auch für Neapolis, dessen südlicher Teil auf einem steilen Hügel liegt. Es ist jedoch nicht zu entscheiden, was für konkrete Anlässe für die Errichtung der beiden termessischen Peripolia bestanden.

Danach verteilte sich das Territorium ( $\pi \varepsilon \rho i \chi \omega \rho \circ \varsigma, \chi \omega \dot{\omega} \rho)$ von Termessos in der

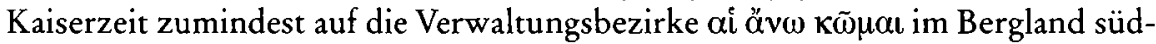
westlich der Stadt (sehr wahrscheinlich die $\kappa \omega ́ \mu \eta$ in Söbüova und das namentlich

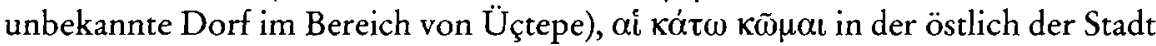
gelegenen Ebene (bezeugt ist $\mathrm{N} \dot{\varepsilon} \alpha \mathrm{K} \omega \dot{\omega} \mu \eta \dot{\eta} \kappa \alpha \dot{\tau} \tau \omega$, vermutlich auch $\mathrm{K} \varepsilon \rho v \alpha v \delta \alpha$,

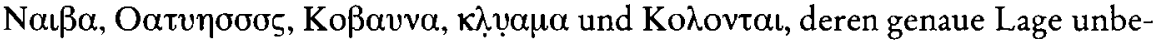
kannt ist) und $\delta \rho v \mu o ́ s$ im nordwestlichen Waldgebiet (?) sowie auf die bisher bekannt gewordenen Peripolia Kelbessos und Neapolis an der Grenze zu Trebenna (und in hellenistischer Zeit vielleicht auch noch auf das namentlich unbekannte Peripolion in TAM III,1, Nr. 1) als Zentren von Lokalgemeinden.

Ein im Jahr 1964 von Hanfmann im Ruinenfeld von Termessos entdecktes und dem Anfang eines Dekrets angehöriges Fragment ${ }^{127}$ zeigt, daß sich Termessos im Jahr 278 v. Chr. mit Sicherheit unter der Herrschaft der Ptolemäer befand ${ }^{128}$, und läßt zugleich fragen, ob sich das termessische Territorium in dieser Zeit im Süden vielleicht bis an die Küste, d.h. bis an den Golf von Antalya, ausgedehnt haben könnte. Diese Frage ergibt sich daraus, daß die Ptolemäer in erster Linie mit den an der Küste befindlichen Städten Kleinasiens Beziehung anknüpften und daß deshalb ein Zugang von Termessos zum Meer zu erwarten wäre. Solch eine Erwartung bereitet uns aber einige Schwierigkeiten: Erstens wurde um 150 v. Chr., d. h. über 100 Jahre nach der ptolemäischen Herrschaft, am Golf von Pamphylien durch den pergamenischen König Attalos II. Philadelphos die Stadt Attaleia gegründet ${ }^{129}$, deren Territorium den termessischen Landbesitz im Osten und teil-

127 L. Robert, Documents de l'Asie Mineure méridionale. Inscriptions, monnaies et geographie (Genève, Paris 1966) 53-58; vgl. dazu auch $M$. Wörrle, Epigraphische Forschungen zur Geschichte Lykiens I, in: Chiron 7 (1977) 47; $E$. Will, Histoire politique du monde hellénistique (323-30 av. JC), I (Nancy 1979) 141 f.; Nollé (o. Anm. 44) 58 f.; G. Hölbl, Geschichte des Ptolemäerreiches: Politik, Ideologie und religiöse Kultur von Alexander dem Großen bis zur römischen Eroberung (Darmstadt 1994) 25, 35, 60.

${ }^{28}$ Das auf einer im fünften Regierungsjahr des ägyptischen Königs Ptolemaios II. Philadelphos (283-246 v. Chr.), im Monat Aủঠvaĩos erfolgten Sitzung der Termessier beschlossene Dekret betrifft die Ehrung für den Makedonen Philippos, Sohn des Alexandros, der vom König zum $\pi \alpha \mu \phi v \lambda$ เá $\rho \chi \eta \zeta$ ernannt worden war. Nach Robert (o. Anm. 127) 54 weist die Erwähnung des Monats Aủovaĩos darauf hin, daß damals in Termessos der makedonische Kalender verwendet wurde.

129 Strab. 14, 667; C. E. Bosch, Studien zur Geschichte Pamphyliens (Ankara 1957) 73 f.; J. Hopp, Untersuchungen zur Geschichte der letzten Attaliden (München 1977) 103 f.: Datierung der Gründung "vermutlich schon ins Jahr 158 v. Chr.“; N. Baydur, Die Münzen von Attaleia in Pamphylien 1, in: JNG 25 (1975) 33-72: die hellenistischen Bronzeprägungen der Stadt schon seit 159 v. Chr.; $A$. H. M. Jones, The cities of the eastern Roman provinces (Amsterdam 21983) 129f.; zu Attaleia vgl. auch W. Ruge, in: RE II,2 (1896) 2156, s.v. „Attaleia, Nr. 3“; Jameson, in: RE Suppl. XII (1970) 110-129, s.v. „Attaleia“; Hellenkemper, Hild (o. Anm. 69) s.v. „Attaleia“. 
weise im Südosten begrenzte. Hatte Termessos, dessen Chora sich vorher tatsächlich bis an die Küste erstreckte, während der Gründungszeit Attaleias einen Teil seines Landbesitzes zugunsten seiner Nachbarstadt verloren? Termessos war 189 v. Chr., d.h. etwa 30 Jahre vor der Gründung Attaleias, durch $\mathrm{Cn}$. Manlius Vulso in das römische Bündnissystem aufgenommen worden, und das möglicherweise während der Regierungszeit des Eumenes II. Soter geschaffene Freundschaftsverhältnis zwischen Pergamon und Termessos wurde durch eine von Attalos II. gestiftete, prächtige Stoa auf der termessischen Agora konsolidiert ${ }^{130}$. Es wäre also undenkbar, daß Termessos zugunsten seines ostsüdöstlichen Nachbarn Boden verloren hätte, zumal Termessos und der Gründer Attaleias in guten freundschaftlichen Beziehungen zueinander standen und noch dazu Termessos und Pergamon Verbündete Roms waren. $10 \mathrm{~km}$ westsüdwestlich von Attaleia lag andererseits vermutlich das seit dem 4. Jahrhundert v. Chr. bekannte Olbia ${ }^{131}$, das wahrscheinlich in der Zeit der großen Kolonisation durch griechische Siedler gegründet wurde, nach der Gründung Attaleias zunächst an Bedeutung und einen Teil seines Landbesitzes zugunsten seiner östlichen Nachbarstadt verlor und schließlich wohl in deren Besitz geriet ${ }^{132}$. Weiter westlich von Olbia, am Sivridağ

130 Vgl. die Architravinschrift des Baues Heberdey, TAM (o. Anm. 11) Nr. 9; vgl. auch ders. (o. Anm. 1) 749; Magie (o. Anm. 3) 264, 280; F. E. Winter, Notes on military architecture in the Termessus region, in: AJA 70/2 (1966) 129; A. Akarca, Yunan Arkeolojisinin Ana Çizgileri I: Şehir ve Savunması (Ankara 21987) 123; K. Bringmann, H. von Steuben, Schenkungen hellenistischer Herrscher an griechische Städte und Heiligtümer: Zeugnisse und Kommentare (Berlin 1995) 377 f., Nr. 303. Zur Politik Attalos' II. in Pisidien vgl. auch R. A. Kearsley, The Milyas and the Attalids: A decree of the city of Olbasa and a new royal letter of the second century B.C., in: AS 44 (1994) 47-57.

131 Heute wahrscheinlich die Ruinen auf einem Felshügel an der Küste, nordöstlich von Hurma (Türkei-Karte [o. Anm. 1] IIc/40: Aşağı/Yukarı Koruma; R. Kiepert, O. Melching, Karte von Kleinasien, Blatt: D II Adalia, Maßstab: 1:400000 [Berlin 21914]: Gurma); Sahin (o. Anm. 72), der Olbia beim heutigen Kemer (Türkei-Karte [0. Anm.1 ] IIe/40) lokalisieren möchte; zu Olbia vgl. Strab. 14, 667; Plin., nat. 5, 96; Ptol., geogr. 5, 5, 2; Stepb. Byz. 489; J. A. Cramer, A geographical and historical description of Asia Minor, II (Oxford 1832, ND: Amsterdam 1971) 274f.; Spratt, Forbes (o. Anm. 64) I 215. 218; Ramsay (o. Anm. 62) 343, Anm. 2; ders., Antiquities of Southern Phrygia and the border lands, in: AJA 4 (1888) 8, Nr. D.5; K. G. Lanckoroński, Städte Pamphyliens und Pisidiens I: Pamphylien (Prag, Wien, Leipzig 1890) 5, Nr. 3; 18; W. Ruge, in: RE XVII,2 (1937) 2404f., s.v. „Olbia, Nr. 2“; Bean (o. Anm. 11) 109-111; F. Imboof-Blumer, Kleinasiatische Münzen, I-II (Wien 1901-1902, ND: Hildesheim, Zürich, New York 1991) 309; Jones (o. Anm. 129) 129 f.; Zgusta (o. Anm. 23) $436 \$ 926-2 ; N$. Çevik, The localization of Olbia on the Gulf of Pamphylia, in: Lykia 1 (1994 [1995]) 89-102; ders., Antalya-Hurma Köyü'nde Bir Çiftlik Yerleşimi, in: Lykia 2 (1995 [1996]) 39-61; ders., Kent Antalya'nın Arkeolojik Envanteri Projesi II: Hurma Köy Yüzey Arasstırmaları, in: AST 14,1 (1996 [1997]) 235-251; Hellenkemper, Hild (o. Anm. 69) s.v. „Olbia“.

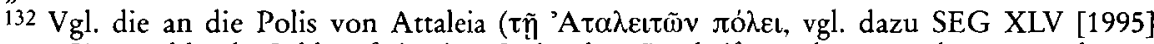
1771C) zu zahlende Geldstrafe in einer Sarkophag-Inschrift aus der ca. $4,5 \mathrm{~km}$ westsüdwestlich von Hurma gelegenen Nekropole einer antiken Siedlung im unmittelbar südöstlichen, fast unzugänglichen Waldgebiet (nahe dem westlichen Hang des Tahragüngediği Dağı [Türkei-Karte (o. Anm. 1) IIc/40], nördlich des Arapuçtu Tepesi [Türkei-Karte (o. Anm. 1) IIc/39]), Çevik, Antalya-Hurma (o. Anm. 131) 44. 46; ders., Kent Antalya'nın (o. Anm. 131) 239. 242. Nach einer Revision im Jahre 2000 konnten wir an derselben Stelle des Textes mit 
beim heutigen Dorf Çăglarca, befand sich schließlich Trebenna ${ }^{133}$, dessen Herrschaftsbereich in Form einer Sympolitie auch das bei Gedeller ${ }^{134}$ am Ende des Gökdere-Tales gelegene Onobara ${ }^{135}$ umfaßte. Angesichts all dieser Belege muß außer Betracht bleiben, daß sich die termessische Chora jemals bis zum Meer ausgedehnt hätte ${ }^{136}$.

Vom Osten und Nordosten der termessichen Chora haben wir oben gesprochen. Was den Norden betrifft, so bestätigt auch eine von uns in Keklicek, $12 \mathrm{~km}$ westnordwestlich von Termessos ${ }^{137}$, gefundene Inschrift ${ }^{138}$ mit der in termessischen Inschriften üblichen Datierung nach dem Probulen, daß die Gegend mit antiken Siedlungsresten zur Chora von Termessos gehörte. Im $8 \mathrm{~km}$ nordwestlich

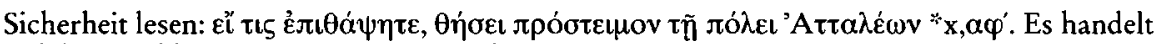
sich hier wohl um eine Kome von Attaleia.

133 Türkei-Karte (o. Anm. 1) IIc/39 (Tramita); für Trebenna vgl. Hier. 679, 7; Lanckoroński (o. Anm. 3) 224, Nr. 184; Paribeni, Romanelli (o. Anm. 5) 203-224; W. Ruge, in: RE VI A,2 (1937) 2268f.; T. R. S. Broughton, Roman Asia Minor, in: T. Frank (Hrsg.), An economic survey of ancient Rome, IV (Baltimore 1938) $734 ; H$. von Aulock, Die Münzprägung des Gordian III und der Tranquillina in Lykien (Tübingen 1974) 53 f., 83 f.; Eyice (o. Anm. 75); G. E. Bean, in: PECS, 932; Jones (o. Anm. 129) 103. 106 ff.; Zgusta (o. Anm. 23) 633 \$1363-2; Mitchell (o. Anm. 9) 77, Anm. 69; N. Çevik, I. Kızgut, S. Aktas, 1997 Yil ${ }_{1}$ Trebenna ve Çevresi Yüzey Araştırmaları, in: AST 16,2 (1998 [1999]) 401-421; Hellenkemper, Hild (o. Anm. 69) s.v. „Trebenna“.

$13418 \mathrm{~km}$ südwestlich von Antalya; Türkei-Karte (o. Anm. 1) IIc/39 (Kedetler).

135 R. Heberdey, E. Kalinka, Bericht über zwei Reisen im südwestlichen Kleinasien (Wien 1897) 36 f., Nr. 47 f.; E. Kalinka, Zur historischen Topographie Lykiens, in: Beiträge zur Alten Geschichte und Geographie. Festschrift für Heinrich Kiepert (Berlin 1898) 168; Kiepert, Melching ([o. Anm. 131] verzeichnet es südöstlich von Gederler [= Gedeller]); Anti (o. Anm. 78) 666 f., 740, 743 (Nr. 5), 747 (Nr. 7), 751 (Nr. 9), 754; Viale (o. Anm. 78) $381 \mathrm{f}$. (Nr. 32); W. Ruge, in: RE XVIII,1 (1939) 485, s.v. „Onobara“; Eyice (o. Anm. 75) 26, Anm. 13; Zgusta (o. Anm. 23) 439 \$936; Çevik, Antalya-Hurma (o. Anm. 131) 46f. (Lokalisierung ohne Beweise in Hurma) = SEG XLV (1995) 1771; Trebenna und Onobara werden

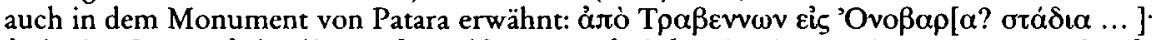

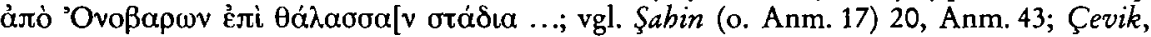
Antalya-Hurma (o. Anm. 131) 50. Auf Grund mehrerer von uns 1998 in einer spätantiken Siedlung im $1 \mathrm{~km}$ südöstlich von Gedeller gelegenen Waldgebiet Deveboynu (Türkei-Karte [o. Anm. 1] IIc/39-40) gefundener Inschriften (erwähnt werden Trebennaten aus Onobara in

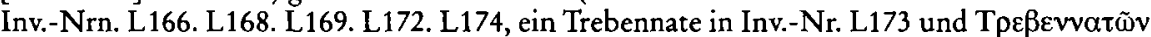
ó $\delta \tilde{\eta} \mu \mathrm{o}$ in Inv.-Nr. L170) vermuten wir, daß dieser Ort das Zentrum von Onobara war, zu dessen Territorium das Gebiet um Gedeller gehörte. Dies würde auch der topographischen Situation im Stadiasmos von Patara entsprechen.

$136 \mathrm{Vgl}$. auch $R$. S. Bagnall, The administration of the Ptolemaic possessions outside Egypt (Leiden 1976) 114. In der sog. Lex Antonia de Termessibus (CIL, 2I, 589; ILS, I, 38) wird $\mathrm{zwar}$ von portoria maritima und insulae gesprochen, was auch an einen Zugang von Termessos zum Meer denken läßt. Da sich jedoch im Golf von Antalya keine nennenswerten Inseln befinden und da ein brauchbarer Hafenplatz in der Nähe von Termessos nicht vorhanden ist, wird im allgemeinen die Ansicht vertreten, daß sich diese lex wohl nicht auf Termessos allein, sondern auf ein größeres Gebiet bezog und daß somit das termessische Territorium nicht bis ans Meer reichte.

$1374 \mathrm{~km}$ östlich von Yazır, $4 \mathrm{~km}$ südöstlich von Bayar; Türkei-Karte (o. Anm. 1) IIa/38 (Keklicikkahvesi); für Keklidschek-Kafe vgl. Heberdey (o. Anm. 4) 11.

138 Unsere Inv.-Nr. T296. 
von Termessos, im Waldgebiet gelegenen Korucuk Mahallesi ${ }^{139}$ von Bayatbademlisi ist ein Meilenstein ${ }^{140}$ erhalten. Auf Grund dieses durch die Polis von Termessos aufgestellten Steines, den wir bei einer Bereisung 1993 wiederfanden und der aus Zeitmangel nicht aufgenommen werden konnte und später verlorenging, und der im $5 \mathrm{~km}$ östlichen Weiler Gedikarası von Bayatbademlisi von uns gefundenen Inschrift ${ }^{141}$, in der mehrere Personen termessischer Herkunft genannt werden, liegt die Gegend innerhalb der termessischen Chora ${ }^{142} .2 \mathrm{~km}$ südwestlich von Korucuk $^{143}$ gibt es eine hellenistisch-römische Ruinenstätte mit einem Turm ${ }^{144}$ und wenigstens vier anderen Gebäuden ${ }^{145}$. Etwa $10 \mathrm{~km}$ nordnordöstlich von Termessos und etwa $6 \mathrm{~km}$ nördlich des Yenice Boğazı, wohl an der Grenze zu Perge, befindet sich in der Höhle Karain am südsüdöstlichen Hang des Şam Dağ ${ }^{146}$ das zur termessischen Chora gehörige Heiligtum der Meter Oreia ${ }^{147}$.

\section{Abkürzungen}

ABSA: $\quad$ Annual of the British School at Athens

ACO: $\quad$ Acta Conciliorum Oecumenicorum

AE: $\quad$ L'Année épigraphique

AJA: American Journal of Archaeology

AMS: $\quad$ Asia Minor Studien

AnzWien: $\quad$ Anzeiger der Österreichischen Akademie der Wissenschaften in Wien,

Arr., anab.: $\quad$ Ahilosophisch-historis

AS: $\quad$ Anatolian Studies

ASAA: Annuario della R. Scuola Archaeologica di Atene e delle Missioni Italiane in Oriente

AST: $\quad$ Araştırma Sonuçlan Toplantısı

BCH: Bulletin de Correspondance Hellénique

BE: $\quad$ Bulletin épigraphique

CIL: $\quad$ Corpus Inscriptionum Latinarum

CRAI: $\quad$ Comptes rendus de l'Académie des Inscriptions et Belles-Lettres

DHGE: Dictionnaire d'histoire et de géographie ecclésiastiques

Dion. Hal., ant.: Dionysios v. Halikarnaß, Antiquitates Romanae

139 Türkei-Karte (o. Anm. 1) IIa/38 (Korucukdam); D. French, 1989 Roma Yolları, Miltaşları ve Yazıtları Araştırmaları, in: AST 8 (1990 [1991]) 233; ders. (o. Anm. 20) 73.

140 French (o. Anm. 20) 73.

141 Unsere Inv.-Nr. T295.

142 Vgl. auch Heberdey (o. Anm. 4) 5, Fig. 1.

143 Türkei-Karte (o. Anm. 1) IIa/38 (Karakol); Spratt, Forbes (o. Anm. 64) Karte; Lanckoroński (o. Anm. 132) Karte; Heberdey (o. Anm. 4) 5, Fig. 1 (antike Mauer und Stätte).

144 Woodward, Ormerod (o. Anm. 24) 83, Nr. 1.

145 French (o. Anm. 20) 73.

146 Etwa $25 \mathrm{~km}$ nordnordwestlich von Antalya; Heberdey (o. Anm. 4) 5, Fig. 1; TürkeiKarte (o. Anm. 1) IIa/39.

147 G. Moretti, Le grotte sacre di Iuvadja, in: ASAA 6/7 (1923/1924 [1926]) 545-554; S. Şahin, Meter Oreia von Karain/Antalya: Eine Grottengöttin in Südkleinasien, in: EA 17 (1991) 126-132; Hellenkemper, Hild (o. Anm. 69) s.v. „Karain“. 
Hesperia: Journal of the American School of Classical Studies at Athens

EA: $\quad$ Epigraphica Anatolica

Eusth., comm.

Dion.: Eustathios, Commentarii ad Dionysium periegetam

Hier:: Hierokles, Synekdemos

Hist. Aug.: Historia Augusta

IGR: $\quad R$. Cagnat, Inscriptiones Graecae ad res Romanas pertinentes, I-IV

(Paris 1911-1927)

IK: Inschriften griechischer Städte aus Kleinasien

ILS:

JHS:

JNG:

JÖAI:

Lykia:

MdI-A:

H. Dessau, Inscriptiones Latinae Selectae, I-III (Berlin 1892-1916)

Journal of Hellenic Studies

Jahrbuch für Numismatik und Geldgeschichte

Jahreshefte des Österreichischen Archäologischen Instituts

Anadolu-Akdeniz Arkeolojisi

Abteilung

MdI-I: $\quad$ Mitteilungen des Deutschen Archäologischen Instituts, Abteilung

Istanbul

MonAL: $\quad$ Monumenti antichi, pubblicati per cura della Reale Accademia dei Lincei

Ncirc: $\quad$ Numismatic Circular

Not. Dign.: $\quad$ O. Seeck, Notitia Dignitatum, accedunt Notitia Urbis Constantinopolitanae et Latercula Prouinciarum (Berlin 1876)

Not. ep.: J. Darrouzés, Notitiae episcopatuum Ecclesiae Constantinopolitanae (Paris 1981)

PECS:

The Princeton Encyclopedia of Classical Sites, ed. R. Stillwell (Princeton 21979)

Plin., nat.: Plinius d. Ä., Naturalis historia

P\&P: $\quad$ Past and Present

Ptol., geogr: $\quad$ Ptolemaios, Geographika

RE: $\quad$ Paulys Realencyclopädie der classischen Altertumswissenschaft

REG: Revue des Études Grecques

SCIV: $\quad$ Studii şi cercetari de istorie veche şi arheologie

SEG: $\quad$ Supplementum Epigraphicum Graecum

SIG ${ }^{3}: \quad$ W. Dittenberger, Sylloge Inscriptionum Graecarum, I-IV (Leipzig ${ }^{3}$ 1915-1924)

Steph. Byz:: Stephanos (v. Byzanz), Ethnika

Strab.: Strabon, Geographika

TAM: Tituli Asiae Minoris

TAVO: $\quad$ Tübinger Atlas des Vorderen Orients

Thuk:: Thukydides

TIB: Tabula Imperii Byzantini

Zos., hist.: Zosimos, Historia nova

ZPE: $\quad$ Zeitschrift für Papyrologie und Epigraphik 
